\title{
La platica de fray Domingo de Santo tomás (1560)
}

La platica de fray Domingo de Santo Tomás (1560)

The platica of fray Domingo de Santo Tomás (1560)

\section{Gerald Taylor}

\section{(2) OpenEdition}

\section{Journals}

Édition électronique

URL : http://journals.openedition.org/bifea/7039

DOI : $10.4000 /$ bifea.7039

ISSN : 2076-5827

Éditeur

Institut Français d'Études Andines

Édition imprimée

Date de publication : 1 décembre 2001

Pagination : 427-453

ISSN : 0303-7495

Référence électronique

Gerald Taylor, "La platica de fray Domingo de Santo tomás (1560) », Bulletin de l'Institut français d'études andines [En ligne], 30 (3) | 2001, mis en ligne le 08 décembre 2001, consulté le 10 décembre 2020. URL : http://journals.openedition.org/bifea/7039; DOI : https://doi.org/10.4000/bifea.7039

Les contenus du Bulletin de l'Institut français d'études andines sont mis à disposition selon les termes de la licence Creative Commons Attribution - Pas d'Utilisation Commerciale - Pas de Modification 4.0 International. 


\title{
LA PLATICA DE FRAY DOMINGO DE SANTO TOMÁS (1560)
}

\author{
Gerald TAYLOR*
}

\begin{abstract}
Résumé
La Platica adressée à tous les Indiens et la formule de la Confession Générale sont les deux seuls témoignages du prêche prétridentin en quechua qui survivent jusqu'à nos jours et ceci grâce à leur incorporation comme échantillons de la langue générale dans la grammaire et le Lexicon composés par Domingo de Santo Tomás et publiés à Valladolid en 1560. Dans cet article, nous étudions la variante de la Langue Générale, véhicule de la Platica, et le lexique religieux élaboré par les premiers missionnaires afin d'expliquer la foi chrétienne aux Indiens. La version normalisée du texte quechua est suivie d'une traduction française annotée. Des annexes reproduisent le texte quechua original avec les gloses espagnoles établies par Santo Tomás, le document espagnol qui a servi de modèle à l'élaboration du texte quechua et la transcription de la Confession Générale, sa reformulation en quechua normalisé et sa traduction française.
\end{abstract}

Mots clés : Quechua, lengua general, évangélisation, lexicologie.

\section{LA PLATICA DE FRAY DOMINGO DE SANTO TOMÁS (1560)}

\section{Resumen}

La Platica para todos los Indios y la fórmula de la confesión general son los únicos dos testimonios de la prédica pretridentina en quechua que se han conservado hasta el día de hoy y ello debido a su incorporación como muestras de la lengua general en la gramática y lexicón compuestos por Domingo de Santo Tomás y publicados en Valladolid en 1560. En este artículo estudiamos la variante de la lengua general utilizada en la Platica y el léxico religioso elaborado por los primeros misioneros para explicar la fe cristiana a los indios. Una traducción francesa comentada sigue a la versión normalizada del texto quechua. Los anexos reproducen el texto quechua original con las glosas castellanas establecidas por Santo Tomás, el documento en castellano que sirvió de modelo para la elaboración del texto quechua y la transcripción de la confesión general, sa reformulación en quechua normalizado y su traducción al francés.

Palabras claves: Quechua, lengua general, evangelización, lexicología.

\section{THE PLATICA OF FRAY DOMINGO DE SANTO TOMÁS (1560)}

\section{Abstract}

The Platica para todos los Indios and the text of the General Confession are the only two examples of missionary writing in Quechua prior to the Council of Trent to come down to us and

${ }^{*}$ CNRS. Instituto Francés de Estudios Andinos, Casilla 18-1217, Perú. 
their survival is due to their having been incorporated in the Grammar and the Lexicon composed by Domingo de Santo Tomás and published in Valladolid in 1560 as samples of the Lengua General. This article analyses the form of the Lengua General in which the Platica was written and the religious vocabulary established by the first missionaries in order to explain the Christian faith to the Indians. An annotated French translation follows the normalised version of the Quechua text. The annexes reproduce the original Quechua text and the Spanish glosses of Santo Tomás, the Spanish document on which the Quechua text was based and the transcription of the General Confession, a version of the same text in normalised Quechua and its French translation.

Key words: Quechua, lengua general, evangelization, lexicology.

La Platica adressée à l'ensemble des Indiens, publiée en 1560 à Valladolid comme appendice à la grammaire de la langue générale des Indiens des royaumes du Pérou (1) de Fray Domingo de Santo Tomás, est le premier texte quechua d'une certaine importance à nous être parvenu. Cette même année, Santo Tomás publie aussi un autre texte, la formule de la Confession Générale en langue générale. Nous reproduisons ici la version normalisée de ces deux documents (2).

D'après Santo Tomás, la langue qu'il décrit dans sa grammaire est la langue véhiculaire de toutes les terres qui avaient été soumises à l'Inca Huayna Capac et que l'on utilisait encore dans tout cet immense territoire ("plus de mille lieues de long et cent de large"). C'était la langue dans laquelle communiquaient tous les nobles et les notables du royaume et une partie importante des gens du commun (3).

À propos de la langue véhiculaire avec laquelle les Espagnols se familiarisèrent pendant les premiers temps de l'époque coloniale, nous avons écrit en 1981(4) :

"La lecture des premiers chroniqueurs de la conquête nous apprend qu'à l'arrivée des Espagnols, une multitude de langues - parmi lesquelles ils classaient sans doute les variantes locales du quechua et de l'aymara se parlaient dans l'empire des Incas mais que la connaissance d'une seule

(1) [...] lengua general de los Indios de los Reynos del Peru.

(2) La Plática a été la base d'un cours sur la langue générale que nous avons donné a partir de 1980 à l'Institut des Hautes Études d'Amérique Latine. Nous avons utilisé l'édition fac-similée du quatrième centenaire de l'UNMSM pour réaliser ce travail. Pour ne pas compliquer notre tâche, nous n'avons pas pris en considération la nouvelle édition préparée par Rodolfo Cerrón-Palomino publiée à Madrid en 1994, car la version originale de notre paléographie et de sa normalisation était déjà rédigée à l'époque de sa parution. Le thème principal de nos recherches est l'établissement du lexique religieux, thème qui n'est pas traité par cet auteur.

(3) [...] es la le(n)gua que se co(n)municaua. y de que se vsaua y vsa, por todo el señorio de aquel gran señor llamado Guayna capa, q(ue) se estie(n)de de por espacio de mas de mil leguas en largo, y mas de cie(n)to en ancho. En toda la qual se vsaua generalmente della de todos los señores y principales de la tierra, y de muy gra $(\mathrm{n})$ parte de la gente commu(n) della (Santo Tomás, Grammatica..., $1951: 11)$.

(4) Notre article, dont le titre original était "Un document quechua inédit de Huarochiri”, n'a été publié en français qu'en 1984 comme document de travail de l'E.R.A. 431 du CNRS. Une traduction espagnole est parue sous le nom de "Lengua general y lenguas particulares en la antigua provincia de Yauyos, Perú" dans la Revista de Indias, n 171, Madrid, 1983. Le nombre impressionant d'erreurs contenues dans cette version a motivé une nouvelle publication révisée dans Revista Andina, n5, Cuzco, 1985. 
langue, la langue impériale des Incas, permettait de se faire comprendre dans tout le pays. Les quelques termes quechuas appris par les conquérants et répandus dans leurs écrits représentent une variante de la langue assez proche de celle décrite dans la première grammaire quechua publiée à Valladolid en 1560 ( $c f$. la sonorisation des occlusives après une nasale : condor, ynga, pamba). Une autre caractéristique de cette lingua franca, qu'ils assimilaient à des degrés différents, est la non représentation graphique de l'uvulaire finale (ou de ses variantes) : $c f$. topa $=/$ tupaq/ (5). $r$ est souvent remplacé par $l$ comme dans le quechua huanca et, au moins d'après la toponymie, dans les anciens parlers de la côte. Il est curieux que ces traits phonétiques, qui ne sont pas typiquement ibériques, caractérisent les premiers termes quechuas introduits en espagnol, souvent réintégrés au lexique quechua en tant qu'hispanismes."

Nous aurions pu rajouter à cette série de traits spécifiques la présence du phonème /sh/, transcrit $\langle\mathrm{x}\rangle$ ( $c f$. Caxamalca) et qui apparait sporadiquement (mais pour certains termes d'une manière exclusive : <xamu-> "venir", <xuti> "nom") dans l'oeuvre de Domingo de Santo Tomás où, cependant, la graphie normative le remplace par $\langle s, \mathrm{ss}\rangle$ dont la prononciation, d'après d'autres témoignages de l'époque, différait de celle de $\langle x\rangle$. Rodolfo Cerrón-Palomino a développé la description de ce qu'il appelle la "langue générale de la côte" (1990) et a publié en 1991 un nouveau document possédant des traits qu'il attribue à cette forme du quechua (1991). Dans un travail encore inédit au moment de la rédaction de cet article, César Itier montre l'expansion de cette variante de la langue à des régions éloignées de la côte comme l'était le territoire sud-andin (2001). Nous ne traiterons pas en détail ici la question de l'existence d'une langue générale de la côte ni de la valeur phonétique qu'il faudrait attribuer au phonème transcrit soit $\langle$ s $>$ soit $<s s>$. Dans la version normalisée des textes que nous présentons ici nous le transcrivons par la graphie conventionnelle de $\langle\hat{\mathrm{s}}\rangle$ sans accorder une préférence à aucune des théories sur la nature exacte de sa réalisation. Il s'agit d'un phonème qui se distingue de $/ \mathrm{s} /$ (transcrit $\langle c ̧\rangle$ ou $\langle\mathrm{z}\rangle$ par Santo Tomás) et qui correspond dans sa distribution à la sifflante palatale /sh/ des dialectes quechuas du centre-nord. Dans notre graphie normalisée, nous représentons aussi le phonème uvulaire /q/ en même temps que nous excluons la palatale rétroflexe */ĉ/ sans qu'aucune évidence fournie par le document original puisse appuyer notre option.

Si, comme la thèse de Juan Carlos Estenssoro (1998) nous le montre, la Platica et la formule de la Confession Générale sont des témoignages de l'oeuvre évangélisatrice de l'église pré-tridentine, la langue dans laquelle ils sont rédigés est la même que celle décrite dans la grammaire de Santo Tomás et qui est la base de la plupart des entrées de son Lexicon. Était-elle également la langue générale de l'empire des Incas ? Nous ne pouvons pas répondre avec certitude à cette question. Ce qui la caractérise (en dehors des traits grammaticaux que nous allons retrouver dans toutes les manifestations de la langue générale des $\mathrm{XVI}^{\mathrm{e}}$ et $\mathrm{XVII}{ }^{\mathrm{e}}$ siècles) est la sonorisation des occlusives à la suite d'une nasale, un système complexe d'accentuation et la représentation minimale des

(5) L'exemple cité ici est peu approprié car, comme nous a fait remarquer C. Itier, "seule la forme tupa est attestée hors de Garcilaso et des auteurs influencés par lui" (communication personnelle, octobre 2000). Le toponyme lima </rimaq/ pourrait le remplacer. 
sons du quechua limitée aux normes orthographiques de l'espagnol de l'époque. Si l'on distingue bien deux sifflantes (que nous transcrivons, à l'instar d'autres descripteurs du quechua, par/s/ et /ŝ/ respectivement), cela est dû à l'existence d'une distinction que maintenait l'orthographe espagnole et où $\langle c ̧, z\rangle$ contrastait avec $\langle$ s, ss $\rangle$. Il n'y a pas de trace dans la graphie de la présence d'une uvulaire (/q/), partiellement indiquée dans les documents du III ${ }^{\mathrm{ème}}$ Concile par l'association des graphies $\langle\mathrm{c}\rangle$ et $\langle\mathrm{qu}\rangle$ aux voyelles ouvertes $\langle\mathrm{O}\rangle$ et $\langle\mathrm{e}\rangle$. Le traitement des voyelles dans Santo Tomás n'obéit pas à des règles semblables et reflète, peut-être, une impression auditive dépendant plus de critères phonétiques que d'une conscience des distinctions phonologiques qui caractérisaient la langue. Effectivement, plusieurs dialectes quechuas, tout en conservant un système trivocalique, tendent à ouvrir les voyelles /i/ et /u/ dans n'importe quel contexte (comme dans certains parlers contemporains de Yauyos et de Ferreñafe) et l'enquêteur habitué à un système pentavocalique pourrait facilement confondre le caractère plus ouvert de ces variantes des phonèmes /i/ et /u/ avec les voyelles espagnoles transcrites respectivement $\langle\mathrm{e}\rangle$ et $\langle 0\rangle$. La description qu'en fait Santo Tomás ne nous aide pas à résoudre ce problème. Son but est d'apaiser les inquiétudes des religieux espagnols, découragés par l'idée d'affronter une langue difficile caractérisée par une prononciation complexe et barbare. La richesse du vocabulaire, les tournures très nuancées, la prononciation douce et agréable de cette langue sont l'objet de ses louanges ; sa vertu principale semble être celle de pouvoir être transcrite facilement en utilisant les lettres de l'alphabet espagnol (6). Les religieux devront apprendre cette langue car des milliers d'âmes sont allées en enfer du fait que ceux qui étaient censés les évangéliser ignoraient leur langue (7).

On pourrait effectivement se demander si la langue générale transcrite par Santo Tomás distinguait une consonne uvulaire de la vélaire notée généralement par $\langle c\rangle$ ou $<q u>$. Les dialectes du nord du Pérou (Chachapoyas et Lamas) et les différentes variétés du quechua équatorien parlées en Amazonie péruvienne, dans les régions andine et amazonienne de l'Équateur et dans le sud de la Colombie, ne font pas cette distinction et possèdent en commun avec la langue générale de Santo Tomás la caractéristique de sonoriser les occlusives à la suite d'une nasale. Sans pouvoir apporter une preuve définitive qui appuyerait la présence de l'uvulaire dans la langue véhiculaire des textes que nous étudions ici, nous l'avons restituée sous la forme $\langle q\rangle$ dans la version normalisée de la Platica et de la Confession Générale. Dans le prologue à son Lexicon, Santo Tomás indique parmi les divers facteurs qui facilitent l'apprentissage de cette

(6) Mi inte(n)to pues principal. S. M. ofresceros este Artezillo, ha sido, para q(ue) por el veays, muy clara y manifiestamente, qua(n) falso es lo que muchos os ha(n) querido persuadir, ser los naturales de los reynos del Peru barbaros, \& indignos de ser tractados con la suauidady libertad q(ue) los demas vasallos vuestros lo son. Lo qual claramente conoscera. V. M. serfalso, si viere por este Arte, la gra(n) policia que esta lengua tiene, la abundancia de vocablos, la conuenie(n)cia q(ue) tienen co(n) las cosas q(ue) significa(n). Las maneras diuersas y curiosas de hablar. El suaue y buen sonido al oydo de la pronunciacio $(\mathrm{n})$ della, la facilidad para escriuirse con nuestros caracteres y letras : Qua $(\mathrm{n})$ facil y dulce sea a la pronunciacio(n) de nuestra lengua (Santo Tomás, Grammatica..., 1951 : 9, 10).

(7) Pero quie(n) supiere la grande y extrema necessidad $q(\mathrm{ue})$ ay en aq(ue)llas prouincias, de la predicacio(n) del Eua(n)gelio : y qua(n)tos millares de animas, se ha(n) ydo y va al infierno, por falta d(e) conoscimie(n)to del, y de las cosas d(e) nuestra sancta fe catholica : por defecto de la le(n)gua, sin la qual no se les puede predicar (Santo Tomás, Grammatica..., 1951 : 15). 
langue, la richesse de son lexique qui permet d'éviter la confusion de l'homophonie et l'absence d'une prononciation "âpre" provoquée par des combinaisons complexes de consonnes, de celles qui se prononcent avec la pointe de la langue ou au fond de la gorge. En ce qui concerne la langue générale des Indiens, aussi bien dans sa prononciation que dans le système graphique choisi pour la transcrire, elle se conforme au modèle de l'espagnol, ce qui rend son apprentissage aisé (8). Ces observations suggèrent l'absence de tout son ne correspondant pas à un symbole de l'orthographe espagnole. Par conséquent, l'uvulaire /q/ n'aurait pas fait partie du système phonologique de la langue générale. Ceci nous semble pourtant peu probable dans le contexte linguistique général du Pérou des premières décennies de l'époque coloniale. Sans pouvoir avancer de preuves de l'existence de ce phonème, il nous parait difficile d'imaginer qu'une langue véhiculaire d'une telle importance, qui avait supplanté dans l'administration ecclésiastique toutes les autres variétés, ait pu être dépourvue de l'un des sons distinctifs caractéristiques de l'ensemble dialectal centre-sud (9). Nous ne savons pas à quelle étape de leur développement les dialectes du nord ont confondu l'occlusive uvulaire avec la vélaire ni à quel moment la pratique du quechua s'est généralisée dans ces régions au détriment des autres langues locales. Il est bien probable que la disparition de l'uvulaire soit due à l'influence de substrats non quechuas (10).

Un proto-phonème que nous n'avons pas pris en considération est l'affriquée palatale rétroflexe /ĉ/. En fait, la langue générale de la Platica fait partie d'un ensemble dialectal bien précis qu'un certain nombre de traits isole du reste du domaine quechua. Sa caractéristique distinctive principale est l'évolution, à partir du système traditionnel de quatre personnes, de suffixes pluriels en voie de grammaticalisation. C'est ainsi que l'on peut parler d'une vraie opposition entre une première personne exclusive et inclusive tandis que, dans le système traditionnel, une opposition fonctionnelle opposait une première personne plurielle (excluant l'interlocuteur) à une quatrième personne de nombre indéfini (incluant obligatoirement le locuteur et l'interlocuteur). Il n'y a pas de traces, dans aucun dialecte de cet ensemble, d'une distinction entre deux affriquées palatales. Si nous avons restitué /ĉ/ pour les documents de Huarochirí, c'est à cause de la présence d'un substrat qui

(8) [...] es facil assi para el aprenderla; como para el pronunciarla, porque las cosas que hazen difficultosas vna le(n)gua son, La equiuacacio(n) de los terminos della, que es, vn termino significar muchas cosas, La pronunciacion aspera de muchas letras consonantes juntas co(n) que se escriue, o pronunciarse en la punta de la lengua, o en la garganta. Todo lo contrario de lo qual tiene esta lengua general de los indios del Peru, porque con ser muy abundante y copiosa de vocablos, tiene muy pocas equiuocaciones de terminos, $Y$ en la pronunciacion y manera de escriuir es muy conforme a la nuestra castellana, $q(\mathrm{ue})$ se profiere, pronuncia, y escriue como ella, y assi se aprendera muy facilmente (Santo Tomás, Lexicon..., $1951:$ 7, 8).

(9) "Cela aurait en effet laissé des traces dans ces dialectes. [D'ailleurs,] si, comme le dit Domingo de Santo Tomás, il n'y a pas d'homophonies, c'est que le contraste/k/q/doit exister" (C. Itier, communication personnelle, octobre 2000).

(10) Apparemment, la fusion de la vélaire avec l'uvulaire caractérisait déjà le quechua du nord au $17^{\text {mè }}$ siècle d'après le témoignage de Diego de Molina qui, non sans humour, maugrée contre ceux qui maltraitent ce qu'il appelle la "gutural" : "Echen en la pisina al gangoso que por faltarle la campanilla le falta el gutural; en andas de muertos lleven al hospital de los leprosos a los chachapoyanos, a los de Quito, Cuenca y Loxa, que, aunque el temor del Inca los apretó para que supiesen la lengua cortesana, se quedaron chinchaysuyos." (Romero, 1928 : 78) 
a certainement dû conserver ce son et qui nous a menéà généraliser ce trait pour l'ensemble des textes de cette région. Mais ici non plus, évidemment, nous n'avons pas de preuves de la non existence de / $\hat{c} /$ dans la langue de la Platica.

Domingo de Santo Tomás est-il l'auteur de la Platica et de la formule de la Confession Générale ? Il est en effet possible qu'il ait contribué à leur rédaction. Cependant, la discipline et la cohérence dialectale qui les caractérisent ne semblent pas correspondre au style de celui qui a rédigé la première grammaire et le premier vocabulaire publiés du quechua, où nous trouvons souvent des entrées lexicales et des représentations graphiques d'origine hybride. Certes, Santo Tomás nous explique que la langue qu'il décrit, utilisée et "générale" dans tout le pays, n'est pas la langue "naturelle" de tous ses habitants et c'est ainsi qu'un grand nombre de termes des parlers régionaux ont été incorporés dans le lexique commun et, comme ceux-ci sont déjà généralement acceptés, il les inclut dans son vocabulaire (11). Cette tolérance n'explique pas pourtant la fréquence des lexèmes que l'on classerait aujourd'hui comme Quechua I et qui apparaissent comme synonymes de leurs équivalents du Quechua II (12). D'autres termes, comme chuzco "quatre" (à côté de tagua) ou tamyani. "pleuvoir" (à côté de parani, à peine cité), distinguent aujourd'hui les dialectes quechua I et II du nord du Mantaro. Comme dans le Manuscrit de Huarochirí, on remplace le terme habituel quechua II pour "travailler" llamcani par l'équivalent quechua I uryani. Llamcani est glosé dans le Lexicon par "atentar. tocando", ce qui correspond à son sens dans le Manuscrit.

Certaines incohérences des travaux linguistiques de Santo Tomás se retrouvent dans la Platica. C'est ainsi que la graphie $<\mathrm{x}>$ ayant la valeur phonétique de [sh] apparaît 6 fois dans le mot <xutioc> (/shutiyuq/) "nommé" et une fois dans <xamurca> (/shamurqa/) "il(s) vin(rent)t". Dans le Lexicon, "venir" se traduit par <xamuni > et tous les dérivés de "nom" ont comme racine lexicale <xuti>. Il s'agit d'une anomalie curieuse car, bien que la distinction entre deux sifflantes soit constante dans l'orthographe, l'opposition se fait généralement entre $\langle c ̧, z\rangle / s /$ et $\langle$ s, ss $>/ \hat{s} /$. Quelle que soit la valeur que l'on donne à ce dernier phonème, nous savons qu'il différait dans sa prononciation de celui noté $\langle\mathrm{x}\rangle$ [sh]. Il y a fluctuation entre l'emploi de $\langle\mathrm{c}\rangle$, $\langle$ s $\rangle$, parfois $\langle\mathrm{h}\rangle$ et $\langle\mathrm{x}\rangle$ dans d'autres mots cités dans la grammaire et le Lexicon, mais dans le cas de <xamuni> et de <xuti>, il n'y a pas de variation. Dans le premier chapitre de la grammaire, qui traite de l'orthographe, Santo Tomás insiste sur la nécessité de ne pas se laisser tromper par la variété de manières de prononcer certains mots dans différentes provinces : les Indiens d'une province disent <xámuy> qui signifie "venir", ceux d'une autre province disent $<$ hámuy> avec le même sens (13). Ceux qui pour Santo Tomás représentent la norme disent - apparemment — <xamuy> [shamuy]. Comment peut-on expliquer cette anomalie dans le texte, par ailleurs si soigné, de la Platica autrement qu'en attribuant à

(11) ... como esta lengua (au(n)que es vsada y general por toda la tierra) no es natural en toda, como esta dicho, estan mezclados con los terminos della: $y$ recebidos, y vsados ya generalmente quasi de todos muchos terminos de prouincias particulares, de los quales ta $(\mathrm{m})$ bie(n) po(n)go yo algunos en el vocabulario, porque assi se vsan comu(n)me(n)te (Santo Tomás, Lexicon..., $1951: 15$ ).

(12) Llacta. o marca ciudad, o pueblo generalmente; huncuni. gui. o quixiani. gui. enfermar generalmente; calçado vxóta, o llanque.

(13) Unos Indios de vne prouincia dize(n) (xámuy) q(ue) significa venir; otros en otra prouincia dizen (hámuy) en la misma significacio(n) (Santo Tomás, Grammatica..., 1951:18). 
Santo Tomás le rôle de rédacteur principal ? Il nous semble que l'explication est fournie par Santo Tomás lui-même quand il nous informe qu'en dépit de l'existence [au Pérou] de beaucoup d'autres langues particulières (presque chaque province possède la sienne), la générale était la langue véhiculaire comprise dans tout le pays, la plus utilisée par les nobles et les notables et une partie importante des autres Indiens. Cette langue n'avait jamais été aussi répandue dans les temps anciens qu'au moment où Santo Tomás rédigeait ses travaux car le contact permanent, les rapports commerciaux et les fréquentations que maintenaient les Indiens entre eux, leurs présence dans les villages et marchés des Chrétiens, leur service auprès des Espagnols et les corvées qu'ils devaient réaliser, établissaient des rapports entre gens de différentes provinces qui, pour communiquer entre eux, étaient obligés d'avoir recours à la langue générale (14). Comme toujours dans un cas analogue, certains éléments empruntés aux dialectes apparentés, ont été assimilés et sont devenus des termes courants. Les religieux, auteurs des textes destinés aux Indiens, ont voulu maintenir une langue relativement uniforme en conservant, au moins dans la graphie, certains traits qui dans les agglomérations de la côte, où résidait une population hétérogène, étaient peut-être en train de devenir caducs. Santo Tomás respectait, aussi bien qu'il le pouvait, ces critères. Cependant, dans le cas de <xamuy> et de <xuti>, termes extrêmement fréquents, peut-être le désir de ne pas trop s'éloigner de la prononciation réelle a-t-il prédominé sur l'obligation d'être fidèle à un modèle linguistique précis (15). S'agit-il donc de corrections apportées par

(14) ... ay en ella otras muchas lenguas particulares, que quasi en cada prouincia ay la suya, pero esta es la general y entendida por toda la tierra, y mas vsada de los señores, y gente principal, y de muy gran parte de los demas Indios, Y nunca esta le(n)gua en los tiempos antiguos fue tan generalmente vsada quasi de todos, como el dia de oy. Porque co(n) la communicacion, tracto, y grangerias que al presente tiene (n) vnos con otros, y concurso en los pueblos de los christianos, y mercados dellos, assi para sus contractaciones, como para el seruicio de los españoles, para ente(n)derse entre si los de diuersas prouincias, vsan desta general (Santo Tomás, Lexicon..., $1951: 8,9)$.

(15) Il nous semble probable que la prononciation la plus répandue dans l'ensemble du pays ait été /sh/, ce que suggère la survie de cette variante dans la plupart des dialectes Quechua I (en dehors, partiellement, du huanca), dans les dialectes mixtes de Yauyos et du Pérou Septentrional et dans presque tous les parlers Quechua II de l'Équateur. Dans les premières chroniques, nous trouvons également des témoignages de l'emploi de /sh/ sous la forme de la graphie $\langle\mathrm{x}\rangle$. Le choix des évangélisateurs de propager la forme $<\mathrm{s}, \mathrm{ss}>[\hat{\mathrm{s}}]$, au moins dans la graphie, suggère un prestige spécial attribué à cette variante, associée — comme elle devait l'être — à la langue des Incas, la langue de la civilisation. Est-ce que nous devrions donc considérer que la sonorisation des occlusives distinguait aussi la langue de l'élite de Cuzco ? Nous avons trop peu d'éléments sur lesquels nous pouvons nous appuyer pour résoudre cette question. Il est, en effet, possible que la langue générale associée aux anciens centres de prestige de l'Empire, qui incluaient Chincha (dont le parler, d'après Morúa, avait été adopté par les Incas comme langue officielle) et le sanctuaire de Pachacamac, différait beaucoup du parler commun des gens du Cuzco (où un substrat aymara était sans doute très important) sans pour autant, au moins dans les premières décennies de l'époque coloniale, perdre son prestige de langue de communication dans un très vaste territoire. Il est peu probable que le rayonnement de cette langue se réduisait à la seule région côtière. La présence de la variante [ŝ] caractérisait peut-être la protolangue Quechua II, dont l'ensemble des dialectes du sud et la langue générale décrite par Santo Tomás sont dérivés. Peu à peu, [ $[\hat{s}]$ devait se perdre — ou en s'assimilant à/s/comme dans les dialectes dits "CuzcoCollao" et d' Ayacucho ou en se transformant en [sh] — . De toute façon, à l'époque de la rédaction des oeuvres linguistiques de Domingo de Santo Tomás, la présence importante à Lima d'Indiens provenant de tous les coins de l'ancien empire incaïque, aurait certainement réintroduit la prononciation [sh] qui 
Santo Tomás à un texte préexistant ?C'est bien possible. D'ailleurs, cela se comprendrait en prenant en considération le fait que, probablement, ces textes reflètent un état de la langue qui n'était déjà plus courant à l'époque de la préparation de la grammaire et du Lexicon, pour sa publication définitive.

Il nous semble probable que la composition de la Platica ait été le fruit d'un travail collectif reflétant les projets d'évangélisation de l'Église coloniale antérieurs aux transformations provoquées par le Concile de Trente. Sa fonction dans la grammaire de Santo Tomás, en dehors du fait qu'elle devait illustrer les règles expliquées dans son livre (16), était également celle de servir de modèle à l'auteur. C'est ainsi que les gloses juxtalinéaires qui accompagnent le texte quechua auraient pu l'aider à établir les règles de syntaxe définies dans la grammaire. Sans doute, différentes copies manuscrites de la Platica devaient circuler, ce qui pourrait expliquer certaines incohérences orthographiques, qui sont d'ailleurs très limitées. Ce qui est remarquable dans ce document, c'est le reflet du travail intellectuel qu'il représente : le condensé de doctrine chrétienne est présenté d'une manière claire et presque poétique, comme un mythe d'origine, sans l'agressivité qui allait distinguer des efforts futurs analogues. Le "nous" inclusif de son discours assimile Espagnols et Indiens. Évidemment, le péché principal des Indiens - l'adoration des huacas, résultat de la tromperie du Démon - est mentionné, mais relativement brièvement. Cependant, l'aspect le plus intéressant de cette oeuvre d'évangélisation est la création lexicale. En dehors de Dios pour représenter le Dieu des Chrétiens qui ne devait pas se confondre avec n'importe quel huaca, même victorieux, cauallo pour nommer le cheval et christiano, obligatoire pour désigner celui qui croit en la divinité de Jésus-Christ, tous les mots clés de la nouvelle religion et les concepts philosophiques sur lesquels elle se fonde sont exprimés en quechua. La plupart de ces termes, trop dangereux, car soit ambigus soit trop explicites, ne devait pas survivre aux transformations réalisées par le IIIème Concile. D'ailleurs, il est intéressant de les analyser par rapport à ce qu'on sait aujourd'hui des religions andines, afin de comprendre comment les ouailles indigènes auraient pu interpréter un discours, sans doute fascinant mais peut-être pas totalement chrétien.

Le texte original de la Platica a certainement dû être composé en espagnol. Il est possible que ce soit le document qui apparaît sur la page de gauche en vis-à-vis du texte quechua. Nous en présentons en appendice sa transcription. Nous signalerons dans les notes les rares variations par rapport à la traduction quechua. Parmi les équivalences établies, probablement les gloses spontanées produites par les premiers aventuriers européens, nous trouvons puma "lion" et llama "mouton". Il est probable que les désignations ethniques, huiracocha pour les Espagnols, yanaruna pour les Noirs, opparuna (/upa runa/) pour les Indiens non péruviens (les Mexicains de l'original) et purunruna pour les Indiens non baptisés de la forêt, représentent, dans le premier cas,

correspondait à toutes les occurances où l'écriture officielle employait la graphie $<\mathrm{s}$, ss $>$. Il est d'ailleurs bien possible que dans le quechua de la capitale coloniale la prononciation [ŝ] - si elle avait effectivement existé — ne représentait plus une forme vivante.

(16) Por tanto me parecio no ser fuera de $p$ (ro)posito sino muy co(n)forme a el, poner aqui al fin del arte el praxis de los preceptos y reglas en el dadas, para $q(\mathrm{ue})$ lo $q(\mathrm{ue})$ el lector ouiere ente(n)dido del arte en la theorica, vea puesto en practica (Santo Tomás, Grammatica..., 1951 : 186). 
une manipulation politique du nom du héros culturel du sud, dans le deuxième, un nom descriptif analogue à celui employé par les Espagnols (negros), dans le troisième, un surnom spontané de type universel (17) et dans le quatrième, probablement, une invention savante rappelant le temps-espace chaotique d'avant l'introduction d'une civilisation basée sur l'ordre. Runa englobe l'ensemble des êtres humains, nous les Espagnols, vous les Indiens péruviens, apprentis chrétiens, les autres, les Indiens "mexicains" qui ont dû arriver avec les aventuriers espagnols provenant de l'Amérique Centrale et les Indiens sauvages. Huiracocha n'est utilisé comme ethnonyme que pour se référer à la langue des Espagnols et ne s'oppose pas à runa. Il n'y a que dans le titre que ce dernier terme soit employé comme désignation apparemment exclusive des Indiens. Causac (/kawsaq/) "(ce qui est) vivant" traduit le concept de créature animée ou, plus spécifiquement, celui d'“animal/non humain”. La création s'organise en hanan(c)pacha (/hanan pacha/) "le temps-espace d'en-haut", c'est-à-dire le ciel ou le paradis, caypacha (/kay pacha/) "ce temps-espace présent, actuel" (où nous nous trouvons) et ucupacha (/uku pacha/) "le temps-espace non visible, intérieur et inférieur". Lorsqu'on évoque les grandes migrations des descendants d'Adam et Ève qui devaient peupler l'ensemble de la terre, celle-ci est désignée comme cay rurinpacha (/kay rurin pacha/). Or, rurin, équivalent dans les dialectes quechua I de $u k u$, ne se trouve pas dans le Lexicon de Santo Tomás. Il devait, cependant, lui être aussi familier que la plupart des autres termes d'origine quechua I qu'il cite, surtout si l'on prend en considération le fait que rurin/lurin entre dans la composition des toponymes ayant le sens de "partie" ou "moitié d'en-bas" par opposition à hanan, "partie" ou "moitié d'en haut". Il est possible que le terme employé par les chroniqueurs pour se référer à la moitié d'en-bas (h)urin soit une modification de ura "en bas" sous l'influence de rurin.

Plusieurs décennies de prêche en quechua ont précédé la publication de la Grammaire et du Lexicon de Santo Tomás. Sans doute la question de la codification d'un lexique chrétien approprié a dû être débattue par les prêcheurs des différents ordres présents sur le sol péruvien, surtout au cours des premiers conciles liméniens et au début de l'enseignement de la langue à l'université de San Marcos. Que plusieurs solutions aient été envisagées pour résoudre ce problème — c'est-à-dire l'adaptation d'un lexique quechua spirituel déjà existant ou l'emprunt à l'espagnol de termes jugés intraduisibles dans le contexte de la culture locale - semble évident si nous analysons la Platica (oeuvre de Santo Tomás ou base préexistante de son étude de la langue). Le lexique de la Platica est déjà établi et appliqué d'une manière logique et précise : par exemple, yachachic seul exprime le concept de "créateur" puisque son rival camac est limité à l'évocation de l'âme. Cependant, le Lexicon est beaucoup moins net dans ses définitions que la Platica et, comme glose de "créer" (criar, hazer de nueuo), nous trouvons camani .gui o yachachini .gui (18), et de "créateur" (criador desta manera) camac. o yachachic (p. 35). Dans les diverses définitions de yachachic/yachacchic (sic) qui apparaissent à

(17) Upa runa, c'est-à-dire des hommes sourds-muets, ceux qui sont semblables dans leur condition de subordonnés mais qui diffèrent de nous du fait de ne pas parler une langue intelligible; $c f$. les formes dérivées d'un terme signifiant "muet" qui, dans la plupart des langues slaves, désignent l'allemand, l'étranger par excellence, le voisin omniprésent maniant un parler incompréhensible.

(18) Santo Tomás, suivant le modèle de Nebrija, présente les gloses des verbes sous la forme de la $1^{\text {ère }}$ personne (terminaison -ni), qu'il fait suivre par la terminaison de la $2^{\text {ème }}$ personne, -ngi. Sauf 
la page 296, la valeur actuelle d' "enseignant" prédomine (ayo, que enseña a otro et doctor que enseña), sens qui se retrouve dans les gloses de la base verbale yachachi(yachachini .gui disciplinar, o enseñar // abezar poner costu(m)bre, dar lectio(n) el maestro // abezar enseñar qualquier cosa generalmente). Le concept d'“enseigner" correspond effectivement à la sémantique de base de yachachi- "faire savoir". L'entrée çayñata, yachachini .gui hazer ymagenes de bulto "fabriquer des statues" avec la forme nominalisée correspondante, çayñata yachachic ymaginero, que las haze "sculpteur, façonneur d'images" (p. 244), exprime une nuance plus proche de celle de "créateur". La racine yacha- traduit essentiellement les concepts de "savoir, apprendre, s'habituer" (p. 297) ou "demeurer, résider" (p. 171). César Itier a réalisé une étude approfondie de l'évolution du terme pacha yachachic à partir d'une proto-racine *ĉa- (Pachacuti, 1993 : 151-163). Ici, nous nous bornerons à ce que pouvait être l'un des sens de yachachidans le monde andin à l'époque de la rédaction de la Platica. Il est bien possible que les ouailles n'aient pas perçu immédiatement le sens de "créer" associé à yachachi- (la création ex nihilo étant un concept étranger à leur mentalité religieuse) et qu'un certain flottement entre "instruire" et "faire, fabriquer" subsistait. Pour ce motif, le verbe, beaucoup plus matériel, rura- "faire" forme constamment un doublet avec yachachi-. Une glose qui nous offre, peut-être, une idée de la distinction entre rura- et yachachiest celle de la p. 138 du Lexicon où formar, reducir se traduit par yachachini .gui, o ñatac, rurani .gui, c'est-à-dire que yachachi- est l'équivalent de "refaire, faire de nouveau". Le yachachic est quelqu'un qui transmet un savoir, une capacité, une existence (puisqu'acquérir des connaissances est se pourvoir des outils essentiels pour "survivre", c'est se fortifier, se "vitaliser", donc exister) ; de la matière brute, il "crée" une image, il refait ce qui existe potentiellement, il l'“anime”, c'est-à-dire qu'il lui permet de fonctionner, de se réaliser. Le choix de yachachi- pour traduire le concept de "créer" a dû être le résultat d'une recherche intellectuelle ardue de la part des évangélisateurs d'avant le III ${ }^{\mathrm{ème}}$ Concile et sa diffusion, de préférence à d'autres termes tels que cama-, a sans doute soulevé une certaine résistance. Son acceptation par les Indiens dans le sens de "créer" a probablement été limitée et a constitué surtout une question, comme pour la plupart des autres termes exprimant les valeurs spirituelles chrétiennes, d'assimilation graduelle et partielle. Son rejet ultérieur par Garcilaso (1963, Primera parte, Libro segundo, capítulo II : 44) nous montre sa fragilité en tant que terme destiné à un public plus vaste que les cercles ecclésiastiques de Lima.

Dans son lexique (p. 40), Santo Tomás présente trois gloses pour exprimer le concept d'“âme" (ánima) : camaquenc, o songo, o camaynin (cette dernière étant corrigée dans la liste des errata, p. 17, comme çamaynin). Nous avons traité ces trois termes dans un article publié en 1976, donc nous ne les mentionnerons que très brièvement ici. La racine cama- (/kama-/) est dérivée probablement d'une proto-base *ka-mu-, dont le sens serait à peu près “orienter l'existence (*ka-) vers un destinataire déterminé". Une évolution ultérieure de ce concept serait "unir deux extrêmes en les comblant" ( $c f$. les gloses signifiant "remplir, occuper d'une manière précise"). Le camaque (/kamaqi/) est la source animante qui transmet la force vitale à un être ou à un

lorsque nous citons textuellement un passage, nous nous limitons à en citer les bases : cama-, yachachi-, etc. 
objet afin qu'il puisse se réaliser. Le suffixe - $n$ final est la marque possessive de la $3^{\text {ème }}$ personne. Nous trouvons dans la Platica l'expression camaquenchic (/kamaqi-nchik/) "notre âme" où la terminaison -nchic indique la $1{ }^{\text {ère }}$ personne inclusive. Songo (/ŝunqu/), malgré la traduction populaire de "coeur", ne s'identifie pas nécessairement au muscle cardiaque (19) mais se réfère plutôt à ce qui est l'essence même d'un être ou d'un objet, une partie invisible, intérieure du corps, siège des émotions. Dans le dialecte de Ferreñafe, il désigne le ventre en tant que "partie noble du corps, où se forment les sentiments". Dans la Platica, et camaque et songo sont employés pour désigner l'âme. Lorsque les deux apparaissent ensemble, songonchic camaquenchic, Santo Tomás les glose respectivement par "nuestras animas (nos âmes), nuestros [e]spiritus (nos esprits)". Le troisième terme, çamaynin (/samay-nin/), marqué comme camaquenc par le suffixe possessif de la $3^{\text {ème }}$ personne, se réfère au "souffle", à l'“esprit". Il n'apparaît pas dans la Platica. En dehors du passage déjà cité où camaque, en apposition à songo, est défini comme "esprit", la glose "esprit" correspond à yuyay (yuyayninchic "nuestro [e]spiritu") dont le sens de base est "exercer une activité mentale" (penser, se souvenir). Ni camaque ni songon'ont eu de suite comme traduction d'“âme" qui, dans la littérature d'évangélisation postérieure, se convertit en anima (20). Cependant, dans les Annotaciones de la lengva quichva qui accompagnent le Catecismo Mayor, on retrouve l'association du camaque et de l'“âme", où le caractère artificiel de la traduction de "créateur" par camac est mise en évidence par une curieuse distinction grammaticale établie entre camac 'participe' (= créateur) et la forme possédée camaque( $n)$ (= âme). Les rédacteurs de la Doctrina christiana remplacent le terme camac dans le Credo par rurac (fabriquant, auteur) afin d'éviter la traduction "inconveniente" de "Dieu, âme du monde" (21). Contrairement aux animaux, les êtres humains, lorsque leur chair (aycha) et leurs os (tullu) meurent, leur âme (songo) et leur esprit (camaque) ne meurent jamais. Les termes songo, dont le sens réel, au moins dans ce contexte, est probablement celui d'“essence", et camaque sont renforcés par l'addition de ucupi cac runanchic qui apparaît dans le texte espagnol comme "este hombre nuestro interior (que acá dentro tenemos)". Nous ignorons l'origine de cette expression, mais elle a dû provoquer une certaine confusion dans l'esprit des ouailles

(19) La traduction de /ŝunqu/ par "coeur" (corazón) a pu être généralisée par les chansons populaires, souvent bilingues, où l'influence de l'espagnol est évidente. L'identité de /ŝunqu/ et de corazón se base sur le rôle analogue qu'ils jouent comme foyer des émotions. Il est cependant vrai que Santo Tomás lui-même traduit coraçon de animal et coraçon de la madera par songo et que l'organe examiné par le devin dans le chapitre 18 du manuscrit de Huarochirí, le sonco, semble bien être un "coeur". Dans de nombreux dialectes actuels, ce même terme représente aussi bien le "coeur" anatomique qu'une partie mal définie de l'intérieur du corps, source des émotions.

(20) C. Itier remarque que "soncco est souvent employé avec anima dans plusieurs textes de catachèse du XVII ${ }^{\text {ème }}$ siècle" (communication personnelle, octobre 2000). En effet, il ne s'agit pas de la traduction d'“âme" mais plutôt d'un terme d'appui qui établit la catégorie sémantique (le domaine de l'esprit, du sentiment et du caché) de l'emprunt anima.

(21) Ruraque(n)man, el Criador. ) adviertase que en el Credo se puso ruraque(n) hazedor, y no camaque. Lo vno porque es equiualente a camaque o camac por criador, como lo declara la Yglesia quando dize. Credo en vnu $(\mathrm{m})$ Deum Patrem omnipotente $(\mathrm{m})$ factore $(\mathrm{m})$ caeli \& terrae. Lo otro por excusar amphibologia porque camaquen ta $(\mathrm{m})$ bien significa el alma, y podria entender el Indio que Dios es alma del mundo, lo qual es inconuenie(n)te grande. En los articulos se puso camac: y bie(n), porq(ue) puesto e (n) participio no tiene esta aequiuocacion. (Doctrina christiana y catecismo para instrvccion de los Indios..., Lima 1584 : f. 77v). 
récemment converties car on rajoutait à l'ensemble des sources censées assurer la survie de l'être animé (l'ombre, le souffle, la puissance animante, le "coeur") un "double" intérieur, donc un élément supplémentaire parmi tous ceux dont l'égarement pourrait provoquer la maladie et, éventuellement, la mort. On pourrait se demander si le terme nuna qui, dans des textes d'évangélisation plus tardifs, évoquait l'âme, n'était pas une dérivation de l'"homme intérieur" par le biais d'une variante dialectale, certainement familière aux descripteurs de la langue générale qui citaient régulièrement les formes "chinchaysuyo" dans les avertissements qui accompagnaient leurs travaux. Il s'agirait dans ce cas-ci d'un néologisme érudit censé exprimer une autre nuance d'ánima (22).

Une catégorie très spéciale d'“âmes”, dépourvues de chair et d'os, semblables au camaquenchic (glosé : "nuestra anima”), au yuyayninchic (glosé : "nuestro [e]spiritu”), est constituée par les "serviteurs" (yanancuna) que Dieu a placés dans le ciel. Ceux-ci sont d'une autre espèce que nous. Ils sont appelés angeles ("anges"). Une partie des anges n'a pas voulu obéir aux commandements de Dieu ; ceux-ci ont été bannis du ciel et se trouvent dans le "monde intérieur-inférieur" (ucupacha). On les connaît en espagnol sous le nom de diablos et en quechua sous celui de mana alli çupay ou çupay mauvais. Cette spécification de mana alli ou "mauvais" est importante car le terme supay, que nous avons analysé ailleurs (1980), n' avait pas encore acquis une connotation totalement négative. Dans la section espagnol-quechua du Lexicon, nous trouvons "Demonio, bueno, o malo, çupay" (p. 99) et, dans la section quechua-espagnol, “çupay, angel, bueno, o malo" (p. 279). Cependant, Santo Tomás n’a pas osé, comme cette dernière traduction semblerait le justifier, appeler les "anges" çupaycuna au lieu du terme peu explicite de yana "serviteur".

Dans la traduction française qui suit la version quechua normalisée, nous avons respecté les gloses de l'original, c'est-à-dire que nous avons traduit camaque par "âme" ou "esprit" selon le cas, yachachi- par "créer", etc. Pour que le lecteur puisse avoir une idée de ce que le public indien auquel la Platica s'adresse eût pu comprendre, nous lui suggérons de garder à l'esprit les commentaires sur le lexique contenus dans cette présentation tout en se souvenant que le degré d'acculturation de la population indigène n'était pas uniforme.

Nous présentons comme documents-annexe : A) le fac-similé du texte quechua de Santo Tomás, qui apparaît dans l'édition de 1560, suivi des gloses espagnoles établies par cet auteur (23) ; B) le fac-similé du texte espagnol, base de la Platica quechua, qui se trouve sur la page de gauche en face du texte quechua dans la publication de 1560 ; C) le texte espagnol original de la Confession Générale, la traduction quechua contemporaine (de Santo Tomás ?), la normalisation du texte quechua et sa traduction en français. Ce dernier document dont l'ambition est plus modeste, ne possède évidemment pas le même intérêt lexicographique qui caractérise la Platica (24), cependant il est important en tant que rare témoignage de la première "langue générale"

(22) Voir les observations de César Itier (Pachacuti, 1993, note $8: 142$ ).

(23) Dans l'original, elles apparaissent comme traductions juxtalinéaires du texte quechua.

(24) On voit cependant qu'il reflète le même modèle linguistique que la Platica et que la traduction des termes chrétiens, par exemple, yanancuna "ses serviteurs" désignant les anges, obéit aux mêmes principes. 
del'évangélisation et comme document indiquant les critères des premiers missionnaires en ce qui concerne les péchés que les Indiens étaient susceptibles de commettre. En effet, ceux-ci sont censés se comporter d'une manière plutôt frivole, aimant le jeu, le bavardage, la boisson et négligeant les tâches qui leur ont été attribuées (par les autorités coloniales ?) ; cependant, les fautes dont ils s'accusent ne semblent pas d'une gravité excessive. Surtout, aucune mention n'est faite de l'adoration des huacas, péché considéré comme la source de toutes les erreurs, mentionné dans la Platica et constamment évoqué dans les guides de confession et les recueils de sermons publiés à la suite du III ${ }^{\text {ème }}$ Concile de Lima. 


\section{LLAPA RUNAKUNAPAQ KUNAŜQA}

Wawqiykuna, churiykuna, llapaykichikta churiysininakta kuyaykichik. Chaypaq Dios ruraqinchikpa kamachikuŝqanta willaŝqaykichik paypa churin paymanta kuyaŝ̀am kanqaykichikpaq. Chayrayku alli uyariwaychik kay ñiŝqayta: ñuqanchik llapa runakuna mana caballokunaŝina, mana llamaŝinachu, mana pumaŝinachu, mana ima [h]ayka kawsaqkunaŝinachu kanchik. Pay caballokuna, pumakuna, ima [h]ayka kawsaqkuna wañuptin aycha ŝunqunwan, siinantin tukuy wañu[n] (1). Ñuqanchik llapa runakuna mana[t]aqmi (2) kayŝina kanchikchu. Wañuptinchik kay aychallanchik tullullanchik sapalla wañun ari. S unqunchik kamaqinchik ukupi kaq runanchik wiñay wiñaypaq mana wañunchu, wiñaypaq kawsanqa. Diospa churin kaqkuna, paypa unanchawan unanchaŝqa kaŝpa, paypa kamachikuŝsanta waqaychaŝpapâ̂ ña wañuptin hananpachaman paypa wasinman rinqa chaypi paywan wiñaypaq ancha kuŝikuŝpa tiyanqanpaq. Mana alli kaq runakuna mana Diospa kamachikuŝqanta huñiqkuna ña wañuptin ukupachaman supaypa wasi[n]man rinqa chaypi ñakarikuŝpa tiyanqanpaq paywan wiñaypaq. Chayŝina kaŝpa alli Diospa kamachikuŝqanta uyarichiŝqaykichik hananpachaman rinqaykichikpaq, mana alli supaymanta qiŝspiŝpa. Nawpa ancha paqariqpi mana hananpacha mana inti mana killa mana quyllur karqachu (3). Mana kay pacha karqachu. Manaraqmi kay pachapi llama, mana lluychu, mana atuq karqachu; mana piŝqu, mana qucha, mana challwa, mana sacha, mana imapaŝ, mana ŝukllapaŝ karqachu. Dios ruraqinchik sapalla wiñaymanta karqa. Pay munaŝqanmanta hananpachakta kaypachakta tukuy ima [h]ayka kaqta paymi rurarqa, llapanta yachachirqapaŝ. Hananpachakta kikinpa yanankunap wasinpaq rurarqa. Intikta punchawta (4) illarinqanpaq rurarqa. Killakta, quyllurwan tutakta illarinqanpaq rurarqa. Kay pachakta ñuqanchik runa kaqkuna kawsanqanchikpaq, tiyanqanchikpaq, rurapuwarqanchik. Wayrakta samanqanchikpaq rurapuwarqanchik. Piŝukta challwakta llapa kawsaqkunaktapaŝ ñuqanchikpaq rurapuwarqanchik. Paykunamanta wakinninta mikunqanchikpaq, wakinninta yanapawanqanchikpaq, wakinninta paykunakta rikuŝpa kuŝikunqanchikpaq rurapuwarqanchikmi. Chay hananpachapi ancha achka yanankunakta yachachirqa ángeles shutiyuqta. Paykuna mana aychayuqchu, mana tulluyuqchu. Kamaqinchikŝina chaqaykuna, yuyayninchikŝina chaykunam-ari. Ñuqanchikmanta ŝuk hamu chaykuna. Kay ñiŝqay yanankunamanta wakinnin allin karqa, Dios ruraqinchipa kamachikuŝqanta huñiŝpa. Paykuna kunan hananpachapi kan, ancha [h]atun apu, ancha kusikuŝpa, ancha imayuq, Dios ruraqinchikwan tiyakun-ari. Kaykuna kunan ángeles shutiyuqmi. Wakinnin ancha mana allim tukurqa, mana Diospa kamachikuŝanta huñiŝpa, ancha huchallikurqa, nanaq Diosta piñachirqa. Chay huchallikuŝsqanrayku paykuna mana alli kaqta Dios ruraqinchik ukupachaman qarqurqa; ninapi (5), asnaqpi, tutayaqpi, huchanta muchuŝpa ñakarikuŝpa wiñay wiñaypaq harqaŝqa tiyanqanpaq

(1) Il est possible qu'il manque un tilde sur le $u$ de la deuxième syllabe de guañuc. Dans ce casci, on pourrait interpréter $g u a \tilde{n} u(n) c$ comme wañun, peut-être plus approprié que wañuq dans ce contexte.

(2) Manaracmi est peut-être un lapsus pour manatacmi, qu'on aurait attendu dans ce contexte.

(3) $\operatorname{karqa}\{\mathbf{q a}\}$ chu.

(4) punchaw $\{\mathbf{k}\}$ ta.

(5) $\operatorname{nina}\{\mathbf{q}\}$ pi. 
qarqumurqa kunankamapaŝ chaypi tiyakun. Chaqay akuyllakuna mana alli supay (ŝimiykichikwan) shutiyuq ñuqaykup viracochap ŝimiyku diablos sh[u]tiyuqmi. Ña Dios ruraqinchik kay ñiŝquy tukuyta puchukaŝpa ŝuk qari Adam shutiyuqta, ŝuk warmiwantaq Eua shutiyuqta yachachirqa rurarqapaŝ. Chaykunamanta llapa runakuna, ñuqayku, qankuna, yanarunakuna, uparunakuna, purunrunakuna, may tiqsisinkama runakunapaŝ miraŝqa kanchik. Pay Adam, pay Eua tiqŝinchik. Kay ñiŝ̀qay ñawpa machukunamanta qatiqin m[a]chuyku (6) miraŝqa kaptin Castilla llaqtaykuman rirqa. Apuski[yki]kuna kay llaqtay[ki]chikman shamurqa. Yanarunap uparunap may tiqŝinkama runakunap machunkuna ŝuk ŝuk llaqtaman rakiŝpa anchurirqa. Ŝ inaŝpalla kayman chayman rakinakuŝpa kay rurinpachakta huntamunchik. Chay mana alli supay ñiŝsqaykuna chay ñawpa machunchikta raykurqa, mana allikta yuyachiŝpa "huchallikuychik" ñiŝpa ñirqa. Ŝ inataqmi chay akuylla mana alli supaykuna chay machunchikta raykuchirqa, ñuqanchiktapaŝ qayantin raykuwanchik. Qa[m]kunaktapaŝ (7) ŝnquykichikpi mana allikta yuyachiŝunkichik (panapaŝ mana rikuriptin) ŝunquykichikpi "wakakta rumikta intikta killakta pachakta muchay" ñiŝunkichik. Chaypaq ancha Dios ruraqinchikta piñachirqankichik. Kunanmanta ama ŝina kankichikchu. Kay huchallikuŝqaykichikmanta llakikuychik Dios apunchikman ŝnquykichikwan chayankichik "a athac, apuymi kanki, ruraqiymi kanki" ñiŝpa, "kunankama wakakta muchaŝpa piñachirqayki; kunanmanta wanaŝaqmi; mana ñataq huchalliŝaqchu. Mana ñataq wakakta muchakuŝaqchu. Qam sapallata muchaŝ́qayki ; qamta tukuyta yalliŝpa kuyaŝqqayki”. S S ina kâ̂pa kawsankichik ; sinnataqmi christiano kaŝpa, ña wañu[p]tiykichik (8), hananpachaman rinkichik, paywan wiñay wiñay tiyanqaykichikpaq.
(6) $\mathbf{m}\{\mathbf{u}\}$ chuyku.
(7) qa $\{\mathbf{n}\}$ kunaktapas .
(8) wañutiykichik. 


\section{SERMON (1) ADRESSÉ À TOUS LES INDIENS (2)}

Mes frères, mes fils (3), je vous aime tous comme mes (propres) fils. C'est pour cela que je vous expliquerai les commandements (4) de Dieu, l'auteur de nos jours (5), afin que vous ses fils, soyez aimés par lui. Pour ce motif, écoutez bien ce que j'ai à vous dire. Nous autres, tout l'ensemble des êtres humains, ne sommes pas comme les chevaux, les lamas (6), les pumas (7) ni comme n'importe quel autre animal (8) car, lorsque ceuxlà (9) - les chevaux, les pumas ou les autres animaux — meurent, aussi bien leur chair que leur âme (10) meurent aussi. Nous autres êtres humains ne sommes pas ainsi. Lorsque nous mourons, il n'y a que notre chair et nos os qui meurent. Notre âme (11), notre esprit (12), notre homme intérieur (13), ne meurent jamais, ils vivront toujours. Ceux qui sont les fils de Dieu, s'ils ont reçu ses sacrements (14) et respecté ses

(1) < kuna- "conseiller, aviser, envoyer un message", traduction habituelle de "sermonner, prêcher un sermon". N'apparaît pas dans le Lexicon.

(2) runakuna traduit le concept d'“Indiens" du titre espagnol. Dans le texte de la Platica, cependant, il se réfère à tout être humain.

(3) Termes d'adresse courants qui se réfèrent aux plus âgés et aux plus jeunes et qui contrastent avec le second emploi de "fils" dans ce même énoncé, qui implique un rapport spirituel.

(4) kamachiŝqa = "ce qui a été ordonné", factitif du verbe kama-, d'un sémantisme très complexe mais dont le sens de base est "occuper complètement un espace déterminé" avec une valeur spécialisée de "transmettre la force vitale ou animante à un être ou à un objet spécifique".

(5) Formule par laquelle nous traduisons ruraqinchik "celui qui nous a faits, fabriqués, façonnés". Cet épithète apparaît régulièrement dans tout le texte en contraste avec la racine yachachi-, employée dans le sens de "créer" mais jamais sous la forme de yachachiq "créateur". La racine rura- "faire" est employée généralement comme un appui pour yachachi- dont le sens insolite de "créer" n'est probablement pas évident pour l'auditeur indien.

(6) Remplace l'espagnol oveja "mouton”. À l'époque, les lamas étaient appelés les “carneros de la tierra" par les Espagnols, tandis qu'aujourd'hui, llama est employé dans le sens de "mouton" dans certains dialectes quechuas comme celui de Chachapoyas.

(7) Remplace l'espagnol león "lion". Dans les récits populaires en quechua, le terme león est souvent employé au lieu de puma.

(8) kawsaq “ce qui vit, l'animé". N'apparaît que rarement dans les textes postérieurs ; le terme animal s'étant répandu dans l'ensemble des dialectes modernes et la composition érudite tawachaki “cuadrupède" n'ayant eu qu'un succès limité.

(9) pay, employé comme déictique, ce qui — comme il est fréquent pour les pronoms de $3^{\text {ème }}$ personne - était probablement sa fonction originale. En tant que forme pronominale, ne se réfère aujourd'hui qu'aux êtres humains.

(10) ŝunqun, leur "coeur", c'est-à-dire : leur essence, le centre de leurs sentiments, une des composantes de l'“alma por la qual viuimos" du Lexicon.

(11) sunqunchik, v. note 10 .

(12) kamaqinchik, l'entrée principale dans le Lexicon dans le sens d'“âme”, traduit ici [e]spíritu, qui renforce le sémantisme général de ŝunqunchik "notre âme".

(13) Traduction littérale des textes espagnol et quechua. Renforce le concept d'âme immortelle — l'homme réel, intérieur et invisible — par contraste avec la chair et les os, les supports apparents, destinés à périr.

(14) Ceux qui sont unanchaŝqa "marqués" (c'est-à-dire "distingués") par son signe, son signal, sa marque = unancha $:$ le baptême. 
commandements, lorsque [leurs corps] mourront (15), [leurs âmes] iront à sa demeure dans le ciel pour y vivre dans la joie éternelle. Les êtres humains mauvais, ceux qui ont rejeté les commandements de Dieu, lorsque [leurs corps] mourront, [leurs âmes] iront en Enfer (16) où se trouve la demeure du Démon (17) et là ils souffriront en sa compagnie les tourments perpétuels. Comme ceci est le cas, je vous ferai connaître les commandements du bon Dieu pour que vous puissiez aller au ciel et vous sauver du mauvais Démon. Anciennement, dans un temps très reculé, à l'époque des origines, ni le ciel, ni le soleil, ni la lune, ni les étoiles n'existaient. Cette terre n'existait pas non plus. Il n'y avait pas encore sur cette terre ni lamas, ni cerfs, ni renards. Il n'y avait pas non plus d'oiseaux, de mer (18), de poissons, d'arbres, rien — de toutes ces choses-là, il n'en existait aucune —. Seul Dieu, l'auteur de nos jours, existait depuis toujours. C'est lui, qui de sa propre volonté a façonné le ciel, la terre et tout ce qui existe ; il a tout créé. Il fit le ciel comme demeure pour ses propres serviteurs (19). Il fit le soleil pour éclairer le jour et la lune et les étoiles pour éclairer la nuit. Il fit la terre pour que nous, les êtres humains, puissions avoir de quoi vivre et un endroit où résider. Il fit l'air pour que nous puissions respirer. Il façonna les oiseaux, les poissons et tous les animaux pour nous. Il en façonna une partie pour nous servir de nourriture, d'autres pour nous aider, d'autres pour qu'en les voyant nous puissions nous réjouir. Là-haut, au ciel, il créa une grande quantité de serviteurs appelés anges. Ceux-ci n'ont pas de chair ni d'os. Ils ressemblent en réalité à notre âme, à notre esprit (20), ils sont d'une autre espèce par rapport à nous. Une partie de ces serviteurs était bonne et respectait les commandements de celui qui nous a façonnés; ils sont maintenant au ciel, de très grands seigneurs, et là ils demeurent auprès de Dieu, l'auteur de nos jours, dans la joie et comblés de tout. Maintenant nous les connaissons sous le nom d'anges. Certains devinrent très mauvais et, n'obéissant pas aux commandements de Dieu, commirent un grand péché (21) et provoquèrent ainsi la colère de Dieu. À cause de ce péché, Dieu, l'auteur de nos jours, expulsa ceux qui étaient mauvais en Enfer; il les expulsa pour que, confinés dans le feu, la puanteur et l'obscurité,

(15) L'emploi du gérondif indiquant un changement d'acteur, s'il ne s'agit pas tout simplement d'une anomalie syntaxique, suggère un contraste entre un sujet mortel (le corps) et un sujet immortel (l'âme) que nous avons restitué dans notre traduction. Ce phénomène se répète dans l'ensemble du texte.

(16) Le temps-espace intérieur ou inférieur, l'espace caché, concept correspondant à l'enfer de la tradition chrétienne mais plutôt éloigné de celui de la demeure des ombres (supay > hupay > upay) des procès d'idolâtries.

(17) supay = "ombre", partie de l'“âme" qui, détachée de ses autres composantes, rôdait autour des lieux qu'elle avait fréquentés pendant la vie avant de rejoindre la demeure des ombres des ancêtres, le supaywasi. On distingue dans le Lexicon les bons et les mauvais supay.

(18) L'original espagnol spécifie qu'il s'agit de la mer.

(19) yana, formellement identique au terme signifiant "noir", se rapporte basiquement à n'importe quel "subordonné". Employé dans le sens de "serviteur" ou "prêtre" lorsqu'il s'agit de la pratique d'un culte, il a été choisi ici pour représenter les "anges".

(20) Ici, kamaqinchik remplace ŝunqunchik comme traduction d'“âme" et yuyayninchik, notre pensée, notre souvenir, notre faculté cognitive en général représente "notre esprit", glosé plus haut par kamaqinchik.

(21) Le sens de base de hucha était celui d'“obligation rituelle" dont la non réalisation constituait une faute grave. C'est ce dernier sens qui a été retenu par les évangélisateurs et les dialectes modernes n'ont conservé que le sens acculturé de "péché" associé à celui de "faute" en général. 
ils soient punis de leur péché et demeurent dans la souffrance pour l'éternité. Ils s'y trouvent jusqu'à présent. Ces scélérats sont connus dans votre langue sous le nom de mauvais supay et dans celle de nous autres Espagnols sous celui de diablos. Lorsque Dieu, l'auteur de nos jours, eut achevé tout cela, il créa et façonna un homme appelé Adam ainsi qu'une femme appelée Ève. Tous les êtres humains, nous autres [Espagnols], vous [les Indiens du Pérou], les Noirs, les Indiens du Mexique, ceux de la forêt, tout l'ensemble des hommes y compris tous ceux qui devront encore naître, jusqu'à la fin du monde, descendons de ces deux-là. Cet [homme], Adam et cette [femme], Ève, sont notre origine. Lorsque les descendants de ces premiers parents, les ancêtres de nous autres Espagnols, se furent multipliés, ils allèrent dans notre pays, la Castille. Vos ancêtres vinrent ici dans votre pays. Les ancêtres des Noirs, des Mexicains, de tous les êtres humains qui peuplent la terre jusqu' aux confins du monde, s'éloignèrent [de la terre d'origine] et se dirigèrent tous vers des pays différents. C'est ainsi qu'en nous séparant les uns des autres et en allant tous vers des régions différentes, nous sommes arrivés à remplir ce monde d'ici-bas (22). Les mauvais démons dont nous avons déjà parlé inspirèrent de mauvaises pensées à nos premiers parents en les incitant à pécher. De la même manière, ces scélérats, ces mauvais démons, tentèrent nos ancêtres, [les descendants de nos premiers parents] et, chaque jour, ils continuent à nous tenter et, quant à vous autres, (bien que vous ne les voyiez jamais), ils suscitent de mauvaises pensées dans vos cœurs en vous invitant à adorer les idoles, les pierres, le soleil, la lune et la terre. En les écoutant, vous avez provoqué la colère de Dieu, l'auteur de nos jours. À partir de maintenant, n'agissez plus de la sorte, repentissez-vous de vos péchés, adressez-vous à Dieu, notre Seigneur, avec votre cœur, en lui disant: “Ah, c'est toi mon Seigneur, c'est toi celui qui m'as fait. Jusqu'à présent, je t'ai mis en colère en adorant les idoles. À partir de maintenant, je me corrigerai, je ne pécherai plus, je n'adorerai plus les idoles (23). Je n'adorerai que toi, je t'aimerai au-dessus de tout autre chose". En faisant cela, vous vivrez [dans la joie ?]; en vous comportant ainsi comme de [vrais] Chrétiens, quand vous mourrez, vous irez au Ciel où vous demeurerez auprès de Lui pour l'éternité.

\section{Références citées}

CERRÓN PALOMINO, R., 1990 - Reconsideración del llamado "quechua costeño. Revista Andina, $\mathbf{n}^{\mathbf{0}} 16$ : 335-386; Cuzco : CBC.

CERRÓN PALOMINO, R., 1991 - Un texto desconocido del quechua costeño (s. XVI). Revista Andina, $\mathbf{n}^{\circ} 18$ : 393-413; Cuzco : CBC.

ESTENSSORO, Juan Carlos, 1998 - Du paganisme à la sainteté. - L'incorporation des Indiens du Pérou au Catholicisme - 1532-1750. Thèse de Doctorat Nouveau Régime en Histoire et Civilisations. EHESS, Paris, 743p..

(22) rurin est l'équivalent dans les dialectes Quechua I de ukun des dialectes Quechua II. Il ne se trouve pas dans le Lexicon. Son emploi ici est insolite. On a peut-être voulu — comme c'est sans doute le cas de nuna - créer une nouvelle expression en ayant recours à un autre groupe dialectal pour se référer à un monde "inférieur" en qualité par rapport au Paradis mais sans pouvoir se confondre avec le ukupacha, domaine du diable. La comparaison avec le texte espagnol suggère que kay rurin pacha pourrait se référer au monde entier.

(23) Le texte espagnol ne mentionne pas l'abandon du culte des idoles. 
GARCILASO DE LA VEGA, Inca, 1963 - Comentarios reales de los incas. B.A.E., t. CXXXIII, Madrid : Atlas.

ITIER, C., 2001 - La propagation de la langue générale dans le sud du Pérou. In : Le savoir, pouvoir des élites dans l'empire espagnol d'Amérique: 63-74; Paris : Centre de recherche sur l'Amérique Espagnole Coloniale, Travaux et Documents $\mathrm{N}^{\circ} 3$. Université de la Sorbonne Nouvelle - Paris III.

III CONCILIO LIMENSE, 1584 - Doctrina Christiana y catecismo para instruccion de los Indios, y de las demas personas, que han de ser enseñadas en nuestra sancta Fé. Con un confessionario, y otras cosas necessarias para los que doctrinan, que se contiene en la pagina siguiente. Compuesto por Auctoridad del Concilio Prouincial, que se celebro en la Ciudad de los Reyes, el año de 1583. Y por la misma traducida en las dos lenguas generales, de este Reyno, Quichua y Aymara; Lima.

ROMERO, C. A. 1929 - Un libro interesante [Transcription des Sermones de la quaresma en lengua quechua por el p. FR. Diego de Molina, de la orden de san francisco, cura de guanuco, etc. Año de 1649., Manuscrit B203 de la Biblioteca Nacional del Perú]. Revista Histórica, IX : 51-87 ; Lima.

SANTO TOMÁS, D. de, 1951[1560] - Grammatica, o arte de la lengua general de los Indios de los reinos del Peru, 207p. ; Lima : UNMSM.

SANTO TOMÁS, D. de, 1951[1560] - Lexicon, o vocabulario de la lengua general del Peru, 374p. ; Lima : UNMSM.

TAYLOR, G., 1976 - Camac, camay et camasca dans le Manuscrit quechua de Huarochirí. Journal de la Société des Américanistes, t. LXIII : 231-244 ; Paris.

TAYLOR, G., 1980 - Supay. Amerindia, 5 : 47-63 ; Paris.

TAYLOR, G., 1984 - Un document quechua inédit de Huarochirí. Document de travail de l'E.R.A. 431 du CNRS ; Paris.

TAYLOR, G., 2000 - Lengua general y lenguas particulares en la antigua provincia de Yauyos, [1983, 1985]. In : Camac, camay y camasca y otros ensayos sobre Huarochirí y Yauyos : $35-69$; Cuzco : CBC-IFEA. Travaux de l'Institut Français d'Études Andines, $\mathrm{N}^{\circ}$ 126.

TAYLOR, G., 2000 - Les sermons des religieux espagnols cités dans la chronique de Guaman Poma de Ayala. Amerindia, 24 : 213-226; Paris.

TAYLOR, G., 2001 - La Platica de la Doctrina Christiana - 1584. Amerindia, 25 : 173-188 ; Paris. 


\section{ANNEXE A}

\section{LLAPA RUNACONAPAC CONASCA ${ }^{1}$}

GVáuquijcòna churijcòna ${ }^{2}$ llappay/quichicta ${ }^{3}$ churijsinacta $^{4}$ còyayquíchic. $^{5}$ / Chaypac $^{6} \operatorname{dios}$ ruraque $(n)$ chicpa $^{7}$ camachi/cusca $(n) \operatorname{ta}^{8}$ villascàyquichic,${ }^{9}$ paypa churinc ${ }^{10}$ / paymànta coyascàm ${ }^{11} \mathrm{ca}(n)$ gayquichicpac. ${ }^{12} /$ Chayràyco, ${ }^{13}$ alli oyariuaychic ${ }^{14}$ cay ñís/cayta,${ }^{15}$ ñoca $(n)$ chic llappa runacòna,${ }^{16}$ màna / cauàllocònasina,${ }^{17}$ màna llàmasinachu ${ }^{18}$ / màna pòmasinachu, ${ }^{19}$ màna yma ayca / cauçacconasinachu ${ }^{20}$ cànchic, ${ }^{21}$ Pay caua-/ llocona, ${ }^{22}$ pomacòna, ${ }^{23}$ yma ayca cauçaccò/na, ${ }^{24}$ guañúptinc,${ }^{25}$ aycha sòngonguan,,${ }^{26} / /$ sinantin ${ }^{27}$ tucuy guañuc. ${ }^{28}$ ñoca $(n)$ chic llappa / runacòna ${ }^{29}$ mànaracmi caysina canchic$/ \mathrm{chu},{ }^{30}$ Guañúptincchic, ${ }^{31}$ cay àychalla $(n)$ chic $^{32} /$ túllullanchic $^{33}$ çàpalla guàñu $(n) \mathrm{c}^{34}$ are, ${ }^{35} \mathrm{Sòn} /$ gonchic $^{36}$ càmaquenchic ${ }^{37}$ úcupi cac rú=/nanchic, ${ }^{38}$ viñay viñaypac ${ }^{39}$ màna guañu $(n) \mathrm{c} / \mathrm{chu},{ }^{40}$ viñaypac cauça $(n)$ ga $^{41}$ Diospa churinc / caccòna, ${ }^{42}$ paypa ona $(n)$ chaguan ${ }^{43}$ ona $(n)$ chasca / caspa, ${ }^{44}$ paypa camachicuscanta ${ }^{45}$ guacay/chaspapas,${ }^{46}$ ña guañuptinc, ${ }^{47}$ hanancpacha $/$ man $^{48}$ paypa guacinman ${ }^{49}$ ringa,$^{50}$ chaypi $^{51} /$ payguan $^{52}$ viñaypac ${ }^{53}$ ancha cussicuspa ${ }^{54} /$ tianganpac, ${ }^{55}$ Mana alli cac runacòna, ${ }^{56} / /$ mana diospa camachicuscanta ${ }^{57}$ huñic $=/$ côna $^{58}{ }^{5}$ na guañuptinc ${ }^{59}$ vcupachaman $^{60}$ çu-/paypa guaciman ${ }^{61}$ ringa ${ }^{62}$ chaypi ñacari-/cuspa ${ }^{63}$ tianganpac ${ }^{64}$ payguan, ${ }^{65}$ viñaypac, ${ }^{66} /$ Chaysina caspa ${ }^{67}$ alli $^{68}$ diospa camachi-/cuscanta ${ }^{69}$ oyarichiscayquichic, ${ }^{70}$ hananc / pachaman $^{71}$ ringayquichicpac, ${ }^{72}$ mana alli / çupaymanta quispispa. ${ }^{73}$ naupa ${ }^{74}$ ancha pa/caricpi, ${ }^{75}$ mana hananc pacha,${ }^{76}$ mana indi,${ }^{77} /$ mana quilla,${ }^{78}$ mana cuyllor ${ }^{79}$ carcacachu $($ sic $),{ }^{80} /$ mana cay pacha carcachu, ${ }^{81}$ manaracmi ${ }^{82} /$ cay pachapi ${ }^{83}$ llama,${ }^{84}$ mana lluychu, ${ }^{85}$ mana / atoc carcachu, ${ }^{86}$ mana pisco,${ }^{87}$ mana cocha,${ }^{88} / /$ mana challua, ${ }^{89}$ mana çacha, ${ }^{90}$ mana yma-/pas, ${ }^{91}$ mana sucllapas carcachu. ${ }^{92}$ Dios rura/que $(n)$ chic $^{93}$ çapalla ${ }^{94}$ viñayma $(n)$ ta carca, ${ }^{95}$ Pay / monascammanta ${ }^{96}$, hana $(n) \mathrm{c}$ pachacta,${ }^{97}$ cay / pachacta,${ }^{98}$ tucuy yma ayca cacta $^{99}$ paymi- $^{100} /$ rurarca $^{101}$ llapa $(n)$ ta yachachircapas ${ }^{102} \operatorname{Hana}(n)$ c / pàchacta, ${ }^{103}$ quiquímpa yananccònap ${ }^{104}$ guà/cimpac ${ }^{105}$ ruràrca. ${ }^{106}$ indicta, ${ }^{107} \mathrm{pu}(n)$ chàocta $(\text { sic })^{108}$ ylla/ringa $(m)$ pac $^{109}$ ruràrca. $^{110}$ quillacta ${ }^{111}$ cuyllorgua $(n)^{112} /$ tòtacta yllarínga $(m)$ pac $^{113}$ ruràrca. ${ }^{114}$ Caypa-/chacta ${ }^{115}$ ñòcanchic runa caccòna ${ }^{116}$ cauçan/ga $(n)$ chícpac, ${ }^{117}$ tianga $(n)$ chicpac, ${ }^{118}$ rurapuàrcan/chic. ${ }^{119}$ Guàyracta,,${ }^{120}$ çama $(n)$ ganchícpac, ${ }^{121}$ rura// puarca $(n)$ chic, ${ }^{122}$ Piscocta $^{123}$ chàlluacta, ${ }^{124}$ llappa / cauçaccònactapas ${ }^{125}$ ñocanchícpac ${ }^{126}$ rura-/puarcanchic. ${ }^{127}$ Paycònama $(n)$ ta $^{128}$ guaquinc/nínta ${ }^{129}$ micunga( $\left.n\right)$ chicpac, ${ }^{130}$ guaquínninta ${ }^{131}$ / yanapauanganchícpac, ${ }^{132}$ guaquincnínta, ${ }^{133}$ paycònacta ${ }^{134}$ ricúspa $^{135}$ cussicunga $(n)$ chicpac, ${ }^{136}$ rurapuàrca $(n)$ chicmi.${ }^{137}$ Chay hana $(n)$ cpàcha-/pi, ${ }^{138}$ àncha àchica yananccònacta ${ }^{139}$ yacha-/chírca ${ }^{140} \mathrm{a}(n)$ geles xutiòcta, ${ }^{141}$ Paycona ${ }^{142}$ mana/aychayocchu,${ }^{143}$ mana tulluyocchu. ${ }^{144} \mathrm{Ca}=/$ maquenchicsina $^{145}$ chacaycòna, ${ }^{146}$ yuyay-/ninchicsina ${ }^{147}$ chaycòna mare ${ }^{148}$ ñocan $=/$ chicmanta $^{149}$ suc hamo ${ }^{150}$ chaycòna, ${ }^{151}$ Cay-// ñiscay yanancònama $(n) \operatorname{ta}^{152}$ guaquincninc ${ }^{153} /$ allin carca,${ }^{154}$ dios ruraque $(n)$ chicpa ${ }^{155}$ camachi/ cuscanta ${ }^{156}$ huñispa, ${ }^{157}$ paycòna ${ }^{158} \operatorname{cona}(n)^{159}$ hana $(n)$ c/pachapi canc, ${ }^{160}$ ancha atun appo, ${ }^{161}$ ancha / cussicuspa, ${ }^{162}$ ancha ymayoc, ${ }^{163}$ dios ruraque $(n) / \operatorname{chicguan}{ }^{164}$ tiacunc mare. ${ }^{165}$ Caycòna ${ }^{166}$ cona $(n)^{167} / \mathrm{a}(n)$ geles xutiocmi ${ }^{168}$. Guaquincnìnc ${ }^{169}$ ancha / mana allim ${ }^{170}$ tucurca, ${ }^{171}$ mana diospa cama/chicusca $(n) \operatorname{ta}^{172}$ huñispa, ${ }^{173}$ ancha hochallicur-/ ca, ${ }^{174}$ nanac diosta piñachirca ${ }^{175}$ Chay hocha/llicusca $(n)$ rayco, ${ }^{176}$ paycona ${ }^{177}$ mana alli cacta $^{178} /$ dios ruraquenchic ${ }^{179}$ vcupachamanc ${ }^{180}$ car/curca, ${ }^{181}$ ninacpi $($ sic $),{ }^{182}$ aznacpi, ${ }^{183}$ 
totayacpi, ${ }^{184}$ ho//chanta mochospa, ${ }^{185}$ ñacaricuspa ${ }^{186}$ viñay / viñaypac ${ }^{187}$ harcasca $^{188}$ tianga $(n)$ pac $^{189}$ carcumur/ca, ${ }^{190}$ conancamapas ${ }^{191}$ chaypi ${ }^{192}$ tiacunc. ${ }^{193}$ / Chacày acuyllacòna ${ }^{194}$ mana alli çupay ${ }^{195}$ (simijquichicgua $\left.(n)\right)^{196}$ xutioc, ${ }^{197}$ ñocàycop ${ }^{198}$ vi/ racóchap simijco, ${ }^{199}$ diablos xitiòcmi $($ sic $){ }^{200}$ ña / dios ruraquenchic ${ }^{201}$ cay ñíscay tucuyta $^{202} /$ puchucàspa, ${ }^{203}$ suc cari Adam xutiòcta, ${ }^{204} /$ suc guarmegua(n) $\operatorname{tac}^{205}$ Eua xutiócta $^{206}$ yacha/chìrca ${ }^{207}$ ruràrcapas, ${ }^{208}$ Chaycônama $(n) \operatorname{ta}^{209}$ llap/pa runacòna, ${ }^{210}$ ñocayco, ${ }^{211}$ cancòna,${ }^{212}$ yànaru/nacòna,${ }^{213}$ òpparunacòna,${ }^{214}$ púrun runacò-/na,${ }^{215}$ may ticssincama $^{216}$ runacònapas $^{217} \mathrm{mi}-/$ rasca canchic, ${ }^{218}$ Pay Adam, ${ }^{219}$ pay Eua ${ }^{220}$ / tícssinchic. ${ }^{221}$ Cay ñiscay ${ }^{222}$ ñaupa macho-/cònama $(n) \operatorname{ta}^{223}$ cateque $(n) \mathrm{c}^{224}$ mochoyco $\left(\right.$ sic ${ }^{225}$ miras $=/ c a$, captinc ${ }^{226}$ Castilla, llactaycoma $(n),{ }^{227}$ rirca,$~^{228} /$ Apposquicòna ${ }^{229}$ cay llactaychîcman $(\text { sic })^{230}$, xa-/múrca, ${ }^{231}$ Yanarúnap ${ }^{232}$ Oppa runap, ${ }^{233}$ may-/ ticssincama ${ }^{234}$ runacònap machoncòna, ${ }^{235}$ / suc suc llactaman, ${ }^{236}$ raquîspa, ${ }^{237}$ anchorírca, ${ }^{238} /$ Sinàspalla ${ }^{239}$ cayma $(n)$ chayma $(n)^{240}$ raquinacús/pa ${ }^{241}$ cay rurinc pàchacta ${ }^{242}$ hondamu $(n)$ chic. $^{243} /$ Chay mana alli çúpay ñiscaycòna,${ }^{244}$ chay / ñaupa machonchicta ${ }^{245}$ raycúrca, ${ }^{246}$ mana / allicta ${ }^{247}$ yuyachíspa, ${ }^{248}$ hochallicuychic, ${ }^{249}$ ñis//pa ñirca, ${ }^{250}$ Sinatacmi ${ }^{251}$ chay acuylla ${ }^{252}$ mana / alli çupaycòna, ${ }^{253}$ chay machonchicta ${ }^{254}$ ray/cochirca. ${ }^{255}$ ñócanchictapas ${ }^{256}$ cayantin $^{257}$ ray/coanchic, ${ }^{258}$ cancònactapas $^{259}$ songoyqui-/chicpi $^{260}$ mana allicta ${ }^{261}$ yuyachissù $(n)$ guichic ${ }^{262}$ ( (panapas mana ricuriptinc) ${ }^{263}$ songoyqui/chicpi, ${ }^{264}$ guacacta, ${ }^{265}$ rúmicta, ${ }^{266}$ indicta, ${ }^{267}$ quilla/cta ${ }^{268}$ pachacta $^{269}$ mòchay, ${ }^{270}$ ñissúnguichic ${ }^{271} /$ Chaypac $^{272}$ ancha dios ruraque $(n) \operatorname{chicta}^{273}$ pi/ñachirca $(n)$ guichic, ${ }^{274}$ Còna $(n) \operatorname{ma}(n) \operatorname{ta}^{275}$ ama sina / canguichicchu, ${ }^{276}$ cay hochallicúscayqui-/chicmanta ${ }^{277}$ llaquicuychic, ${ }^{278}$ dios appon/ chicman ${ }^{279}$ songoyquichicguan ${ }^{280}$ chayan-//guichic. ${ }^{281} \mathrm{~A}$ athac appoymi cangui, ${ }^{282}$ rura/ queymi cangui ${ }^{283}$ ñispa,${ }^{284} \mathrm{Cona}(n)$ cama $^{285}$ gua/cacta mochaspa ${ }^{286}$ piñachircayqui, ${ }^{287}$ Cona $(n) /$ manta $^{288}$ guanassácmi ${ }^{289}$ mana ñatac ${ }^{290}$ hocha/llicussacchu, ${ }^{291}$ mana ñatac guacacta ${ }^{292} \mathrm{mo} /$ chacussacchu, ${ }^{293}$ Cam çapallata ${ }^{294}$ mochas-/cayqui, ${ }^{295}$ camta $^{296}$ tucuyta yallispa, ${ }^{297}$ coyas-/cayqui. ${ }^{298}$ Sina caspa, ${ }^{299}$ cauçanguichic, ${ }^{300}$ sina/tacmi ${ }^{301}$ christiano caspa ${ }^{302}$ ña guañutijqui $=/$ chic,${ }^{303}$ hanan pachaman ringuichic, ${ }^{304}$ pay-/guan. ${ }^{305}$ viñay viñay $^{306}$ tiangayquichicpac ${ }^{307} / / /$

\section{GLOSAS DE LA PLÁTICA DE SANTO TOMÁS}

${ }^{1}$ Para todos los Indios consejo| ${ }^{2} \mid$ Hermanos mios y hijos mios $\left|{ }^{3}\right|$ a todos vosotros $\mid$ ${ }^{4} \mid$ a hijos como ${ }^{5} \mid$ os amo. $\mid{ }^{6}$ Por tanto $\left.\right|^{7} \mid$ de dios nuestro hazedor ${ }^{8} \mid \operatorname{los} \operatorname{ma}(n) \operatorname{damie}(n)$ tos $\mid$ ${ }^{9} \mid$ os dire, $\left|{ }^{10}\right|$ del hijos $\left|{ }^{11}\right|$ del amados $\left|{ }^{12}\right|$ para que seays $\left|{ }^{13}\right|$ Por esso| ${ }^{14} \mid$ bien me oyd| ${ }^{15}$ |esto $\mathrm{q}(u e)$ os digo. $\left.\right|^{16} \mid$ Nosotros todos los ho( $m$ )bres. $\left.\right|^{17} \mid$ no cauallos como, ${ }^{18} \mid$ ni ouejas como, ${ }^{19} \mid$ ni leones como, ${ }^{20} \mid$ ni las demas cosas que biuen assi como, ${ }^{21} \mid$ somos. $\left.\right|^{22} \mid$ Los cauallos.| ${ }^{23} \mid$ los leones| ${ }^{24} \mid \mathrm{y}$ las demas cosas q(ue) biuen| ${ }^{25} \mid$ quando muere $\left.(n)\right|^{26}$ la carne con el anima $\left|{ }^{27}\right|$ juntame $(n)$ te ${ }^{28} \mid$ todo muere. $\left|{ }^{29}\right|$ nosotros todos los ho $(m)$ bres $\left|{ }^{30}\right|$ no assi somos,| ${ }^{31} \mid$ quando morimos| ${ }^{32}$ |esta. nuestra carne| ${ }^{33} \mid$ nuestros huessos| ${ }^{34} \mid$ solame $(n)$ te mueren| ${ }^{35} \mid$ si. $\left|{ }^{36}\right|$ Nuestras animas ${ }^{37} \mid$ nuestros spiritus $\left|{ }^{38}\right|$ este de de $(n)$ tro ho $(m)$ bre nuestro| ${ }^{39} \mid$ para siempre jamas, $\left|{ }^{40}\right|$ no muere, $\left|{ }^{41}\right|$ para siempre biuira| ${ }^{42} \mid$ De dios hijos los que son| ${ }^{43} \mid \mathrm{del}$ $\operatorname{co}(n)$ sus sacrame $(n)$ tos $\left.\right|^{44} \mid$ señalados siendo| ${ }^{45} \mid$ del los mandamientos $\left.\right|^{46} \mid$ guarda $(n)$ do los va ${ }^{47} \mid$ quando muere $\left.(n)\right|^{48} \mid$ arriba al cielo ${ }^{49} \mid$ del que es morada $\left|{ }^{50}\right|$ yran $\left|{ }^{51}\right|$ alli $\left|{ }^{52}\right|$ con el| $\left|{ }^{53}\right|$ para sie $(m)$ pre ${ }^{54} \mid$ mucho holga $(n)$ dose ${ }^{55} \mid$ para estar ${ }^{56} \mid$ los malos hombres ${ }^{57} \mid$ que no de dios los mandamientos ${ }^{58} \mid$ guardan, $\left|{ }^{59}\right|$ ya quando mueren| ${ }^{60}$ abaxo de la tierra| ${ }^{61} \mid$ del 
diablo a la casa| ${ }^{62} \mid$ yran, $\left|{ }^{63}\right|$ alli padescie $(n)$ do $\left|{ }^{64}\right|$ estaran $\left|{ }^{66}\right|$ para siempre $\left|{ }^{65}\right|$ con el. $\left|{ }^{67}\right|$ Assi pues siendo, $\left|{ }^{68}\right|$ bien $\left|{ }^{69}\right|$ de dios los mandamientos $\left|{ }^{70}\right|$ os quiera dar a entender ${ }^{71} \mid$ al cielo $\mid$ ${ }^{72} \mid$ para que vays, ${ }^{73} \mid$ del diablo librandoos. ${ }^{74} \mid$ Primero| ${ }^{75} \mid$ muy mucho $t($ iem $)$ po ha. $\left.\right|^{76} \mid$ no cielo, ${ }^{77} \mid$ no sol. $\left|{ }^{78}\right|$ ni luna, $\left|{ }^{79}\right|$ ni estrellas $\mid{ }^{80}$ auia. $\left.\right|^{81} \mid$ ni este mundo auia,| ${ }^{82} \mid$ ni| ${ }^{83} \mid$ en esta tierra| ${ }^{84} \mid$ ouejas, $\left|{ }^{85}\right|$ ni venados $\left|{ }^{86}\right|$ ni zorras auia| ${ }^{87} \mid$ ni aues $\left|{ }^{88}\right|$ ni mar| ${ }^{89} \mid$ ni pezes, $\left|{ }^{90}\right|$ ni arboles, ${ }^{91} \mid$ ni otra| ${ }^{92} \mid$ ni vna au $(n)$ sola cosa auia. $\left|{ }^{93}\right|$ Dios criador nuestro| ${ }^{94} \mid \operatorname{solame}(n)$ te| ${ }^{95} \mid$ sin principio. auia| ${ }^{96} \mid \mathrm{Y}$ qua $(n)$ do fue su voluntad, $\left|{ }^{97}\right|$ el cielo, $\left|{ }^{98}\right|$ este mundo, $\left|{ }^{99}\right| \mathrm{y}$ todo quanto ay| ${ }^{100}|\mathrm{el}|{ }^{101}$ |lo hizo| ${ }^{102} \mid$ todo, lo crio y| ${ }^{103} \mid \mathrm{Al}$ cielo| ${ }^{104} \mid$ del mismo de los criados ${ }^{105} \mid$ para morada| ${ }^{106}|\mathrm{lo} \mathrm{hizo,}|{ }^{107} \mid \mathrm{Al}$ sol| ${ }^{108} \mid$ al dia| ${ }^{109} \mid$ para alumbrar ${ }^{110} \mid$ lo hizo. $\left.\right|^{111} \mid$ A la luna| ${ }^{112} \mid \operatorname{co}(n)$ las estrellas. $\left.\right|^{113} \mid$ la noche para alumbrar $\left|{ }^{114}\right|$ las hizo, $\left|{ }^{115}\right|$ Este mu $(n)$ do $\left|{ }^{116}\right|$ nosotros los hombres| ${ }^{117}$ |para biuir,| ${ }^{118}$ |y para morar, ${ }^{119}$ |nos lo crio.| ${ }^{120}$ |Al ayre| ${ }^{121}$ |para que respirassemos ${ }^{122} \mid$ nos los crio| ${ }^{123} \mid$ Las aues, $\left|{ }^{124}\right|$ los pexes, $\left|{ }^{125}\right|$ todo lo que biue y| ${ }^{126} \mid$ para nosotros ${ }^{127} \mid$ nos lo crio,| ${ }^{128} \mid$ Dellos $\mid{ }^{129}$ |algunas cosas $\mid{ }^{130}$ |para que comamos, ${ }^{131}$ |otras| ${ }^{132}$ |para que nos ayuden y siruan,| ${ }^{133}$ |otras| ${ }^{134}$ |a ellas| ${ }^{135}$ |viendolas,| ${ }^{136}$ |para que nos holguemos, $\left|{ }^{137}\right|$ nos las crio. $\left.\right|^{138} \mid$ Alla arriba en el cielo, $\left|{ }^{139}\right|$ muy muchos criados $\left|{ }^{140}\right|$ crio y hizo, ${ }^{141} \mid \mathrm{q}($ ue $)$ angeles se llaman| ${ }^{142} \mid$ Los quales| ${ }^{143}$ no tienen carne, $\mid{ }^{144}$ |ni huessos.| ${ }^{145}$ |Nuestras animas assi como| ${ }^{146}$ |aquellos, $\mid{ }^{147}$ |nuestro [e]spiritu y assi son| ${ }^{148}$ |aquellos si, ${ }^{149} \mid$ que nosotros son, ${ }^{150} \mid \mathrm{d}(e)$ otra [e]specie| ${ }^{151}$ |aquellos. ${ }^{152} \mid$ Destos q(ue) he dicho criados, ${ }^{153} \mid$ vnos $\left.\right|^{154} \mid$ buenos fuero $(n)\left|{ }^{155}\right|$ de dios nuestro criador $\left|{ }^{156}\right| \operatorname{los}$ ma( $\left.n\right)$ damientos ${ }^{157}$ |obedecie( $n$ )do| ${ }^{158}$ |Ellos| ${ }^{159}$ |agora| ${ }^{160}$ en el cielo estan,| ${ }^{161}$ muy gra $(n)$ des señores.| ${ }^{162} \mid$ mucho alegrandose $\left|{ }^{163}\right|$ muy ricos $\left|{ }^{164}\right| \operatorname{con}$ dios nuestro criador $\left|{ }^{165}\right|$ estan, si.| ${ }^{166} \mid$ Estos $\mid$ ${ }^{167}$ |agora| ${ }^{168}$ |angeles se llaman.| ${ }^{169} \mid$ Otros $\left|{ }^{170}\right|$ muy malos $\left|{ }^{171}\right|$ se tornaro( $\left.n\right),\left|{ }^{172}\right|$ no de dios los mandamie $(n)$ tos $\left|{ }^{173}\right|$ obedecie $(n)$ do $\left|{ }^{174}\right|$ mucho peccaron, $\left|{ }^{175}\right|$ mucho a dios enojaron| ${ }^{176} \mid$ destos peccados por causa, $\left|{ }^{177}\right|$ a ellos $\left|{ }^{178}\right|$ que eran malos, $\left|{ }^{179}\right|$ dios nuestro criador $\left|{ }^{180}\right|$ al infierno| ${ }^{181} \mid$ los hecho| ${ }^{182} \mid$ en el fuego, ${ }^{183} \mid$ en hedor, $\left|{ }^{184}\right|$ en tinieblas ${ }^{189} \mid \mathrm{p}($ ar $)$ a $\mathrm{q}($ ue $)+\mid$ ${ }^{185}$ |por sus pec $(a d)$ os pagando. ${ }^{186} \mid$ y padesciendo| ${ }^{187} \mid$ para siempre| ${ }^{188} \mid$ encerrados $\left|{ }^{189}\right|+$ esten| ${ }^{190} \mid$ los desterro.| ${ }^{191}$ |aun hasta agora| ${ }^{192}$ |alli| ${ }^{193}$ |estan.| ${ }^{194}$ |Yaquellos vellacos| ${ }^{195} \mid$ (mana alli çupay)| ${ }^{196}$ en vuestra le( $n$ )gua| ${ }^{197}$ |se llaman| ${ }^{198}$ |en nuestra| ${ }^{199} \mid$ de los christianos le (n)gua| ${ }^{200}$ |diablos se llaman. ${ }^{201} \mid \mathrm{Ya} \mathrm{q}(u e)$ dios nuestro criador| ${ }^{202}$ |ello q(ue) he dicho todo ${ }^{203}$ |auie(n)dolo acabado. ${ }^{204} \mid \operatorname{Vn}$ varo(n) Adam llamado| ${ }^{205} \mid$ y vna muger| ${ }^{206}$ |Eua llamada| ${ }^{207}$ |crio| ${ }^{208}$ |y hizo:| ${ }^{209}$ Destos| ${ }^{210}$ |todos los hombres.| ${ }^{211}$ |Nosotros,| ${ }^{212} \mid$ vosotros, ${ }^{213} \mid$ los negros, ${ }^{214} \mid \operatorname{los}$ indios d(e) Mexico, ${ }^{215} \mid$ los de los montes, | ${ }^{216} \mid \mathrm{y}$ hasta el fin del mu(n)do| ${ }^{217} \mid$ los ho(m)bres ${ }^{218} \mid$ multiplicados somos.| ${ }^{219} \mid \mathrm{Aq}(u e) 1$ Adam ${ }^{220} \mid \mathrm{aq}($ ue $)$ lla Eua| ${ }^{221}$ |son $\mathrm{n}($ uest $)$ ro $\mathrm{p}($ ri $)$ ncipio. ${ }^{222} \mid$ Destos $\mathrm{q}($ ue $)$ digo| ${ }^{223} \mid$ primeros padres| ${ }^{224} \mid \operatorname{los} \mathrm{q}(u e)$ se siguiero $\left.(n)\right|^{225} \mid$ nuestros padres ${ }^{226} \mid$ multiplicados sie $(n)$ do, $\left.\right|^{227} \mid$ a Castilla. $\mathrm{q}($ ue $)$ es nuestra tierra. $\left.\right|^{228} \mid$ fueron ${ }^{229} \mid \mathrm{V}($ uest $)$ ros antepassados ${ }^{230} \mid \mathrm{a}$ esta vuestra tierra| ${ }^{231}$ |viniero(n). $\left.\right|^{232} \mid$ Los d $(e)$ los negros. ${ }^{233} \mid \mathrm{y}$ indios d $(e)$ Mexico. ${ }^{234} \mid \mathrm{y}$ hasta el cabo d $(e) 1$ $\left.\operatorname{mu}(n) \mathrm{do}\right|^{235} \mid \mathrm{de}$ los ho $(m)$ bres los antepassados ${ }^{236} \mid$ a vna y otra tierra| ${ }^{237} \mid \operatorname{se}$ repartiero $(n)$ :| ${ }^{238} \mid$ y dividiero $\left.(n)\right|^{239} \mid \mathrm{Y}$ assi $\left|{ }^{240}\right|$ a vnas y a otras tierras $\left|{ }^{241}\right|$ diuidiendose. $\left|{ }^{242}\right|$ este mundo| ${ }^{243}$ |henchimos.| ${ }^{244}$ Estos malos demonios $\mathrm{q}(u e)$ os he dicho ${ }^{245} \mid \mathrm{a}$ aq $(u e)$ llos $\mathrm{p}($ ri $)$ meros nuestros padres $\left|{ }^{246}\right| \operatorname{diero}(n)$ occasio $(n)\left|{ }^{247}\right|$ malas cosas ${ }^{248} \mid$ trayendolas a la memoria| ${ }^{249} \mid$ peccad $\left|{ }^{250}\right| \operatorname{dizie}(n)$ doles les dixero $(n) .\left|{ }^{251}\right|$ y assi $\left|{ }^{252}\right|$ estos vellacos $\left|{ }^{253}\right| \mathrm{y}$ malos demonios ${ }^{254}$ |a estos $\mathrm{n}$ (uest)ros $\mathrm{p}$ (ri)meros padres| ${ }^{255}$ |ocasionaron,| ${ }^{256} \mid \mathrm{Y}$ a nosotros| ${ }^{257}$ |cada dia| ${ }^{258} \mid \operatorname{nos} \mathrm{da}(n)$ ocasio(n) d(e) pecar.| ${ }^{259} \mid \mathrm{y}$ a vosotros ${ }^{260} \mid$ en vuestros coraçones $\left|{ }^{261}\right|$ malas cosas| ${ }^{262}$ os hazen pensar, ${ }^{263}$ (aunque ellos no parescen)| ${ }^{264}$ en vuestros coraçones| 
${ }^{265} \mid$ los ydolos, ${ }^{266} \mid$ las piedras, $\left|{ }^{267}\right|$ al sol. $\left|{ }^{268}\right|$ a la luna. ${ }^{269} \mid$ a la tierra| ${ }^{270} \mid$ adorad| ${ }^{271}$ |os dizen,| ${ }^{272}$ Por esso| ${ }^{273}$ |mucho a dios nuestro criador| ${ }^{274}$ |aueis enojado,| ${ }^{275} \operatorname{Do}(n)$ de aqui $\operatorname{adela}(n)$ te $\left.\right|^{276} \mid$ no assi seays, $\left.\right|^{277} \mid$ de estos vuestros peccados $\left.\right|^{278} \mid$ os pese, $\left|{ }^{279}\right|$ a dios nuestro criador| ${ }^{280}$ |con vuestro coraçon| ${ }^{281} \mid$ os llegad.| ${ }^{282} \mid \mathrm{O}$. señor mio soys, $\left.\right|^{283} \mid$ criador mio soys,| ${ }^{284} \mid$ diziendo. ${ }^{285} \mid$ Hasta agora| ${ }^{286} \mid$ a los ydolos adora $(n)$ do, $\left.\right|^{287} \mid$ os he enojado. ${ }^{288} \mid$ De aqui adelante $\left.\right|^{289} \mid$ me emendare|. $\left.\right|^{290} \mid$ no otra vez ${ }^{291}$ |pecare. $\left.\right|^{292} \mid$ no mas a los ydolos $\left.\right|^{293} \mid$ adorare.| ${ }^{294} \mid$ A vos solo| ${ }^{295} \mid$ adorare. ${ }^{296} \mid \mathrm{y}$ a vos $\left|{ }^{297}\right|$ mas q(ue) todas las cosas| ${ }^{298} \mid$ amare, ${ }^{299} \mid$ Assi siendo ${ }^{300}$ |biuireys. $\left.\right|^{301} \mid \mathrm{y}$ assi $\left.\right|^{302} \mid$ christianos siendo. $\left.\right|^{303} \mid$ qua $(n)$ do murieredes $\left.\right|^{304} \mid$ al cielo yreys ${ }^{305}$ |con el.| ${ }^{306}$ |para siempre jamas| ${ }^{307}$ |estar alla.| Amen. 


\title{
ANNEXE B
}

\section{Platica para to-}

\author{
/dos los Indios
}

Hermanos \& hijos mios, a / todos vosotros os amo y / quiero mucho, como a mis / proprios hijos, por tanto os / quiero dezir los ma(n)damien-/tos $\mathrm{d}(e)$ dios, para $\mathrm{q}(u e)$ seays sus hi/jos y amigos suyos. Por esso / estad atentos, y oydme bien / esto que os quiero dezir. Noso-/tros todos los ho $(m)$ bres, no so-/mos como los cauallos, ni co/mo las ouejas, ni como los / leones, ni como las demas co/sas biuas, Porq(ue) los cauallos, / los leones, y todas las otras cosas / que biue $(n)$, quando mue/ren, el cuerpo y el anima // todo juntamente muere, pe-/ro nosotros los ho $(m)$ bres no so/mos assi, q $(u e)$ qua $(n)$ do morimos, / nosotros, y vamos deste mu(n)-/do, solame(n)te muere $\mathrm{n}($ ues $)$ tro cuer/po. Mas nuestra anima y [e]spi-/ritu, este hombre nuestro in-/terior (q(ue) aca de(n)tro tenemos,) / nu(n)ca muere, para siempre ja-/mas biue. Y los que son hi-/jos de Dios (por sus sacrame $(n) /$ tos) y son buenos y guardan / sus mandamientos, van alla / al cielo (que es la morada de / Dios) a donde estarán con el / en muy gran gozo, gloria y / alegria, descanso, y recrea-/cion para siempre jamas. Los / que fueren peccadores y ma-//los y no obedece $(n)$ ni guardan / sus ma $(n)$ damie $(n)$ tos, qua $(n)$ do mue/re $(n)$, sus a $(n) \mathrm{i}(m)$ as $\operatorname{ira}(n)$ al infierno $(\mathrm{q}(u e)$ / es la casa y morada $\mathrm{d}(e) \operatorname{los} \mathrm{d}(e) \mathrm{mo} /$ nios) y alli estaran para $\operatorname{sie}(m)$ pre / pena $(n)$ do. Y pues ha de ser assi, / que las animas d $(e)$ los buenos, / despues que mueren, han de / yr al cielo, a tener gran glo-/ria con dios, y las de los ma-/los co(n) el demonio al infierno / $\operatorname{co}(n)$ pena para sie $(m)$ pre. Oydme / bie $(n)$ esto q $(u e)$ os quiero dezir, $\mathrm{pa} / \mathrm{ra} \mathrm{q}(u e)$ vays al cielo, escapando/os del infierno. Primero mu/cho tiempo ha, no auia cie-/lo, ni sol, ni luna, ni estrellas, / ni auia este mundo inferior, / ni en el auia ouejas, ni vena-/dos, ni zorras, ni aues, ni mar, // ni pexes, ni arboles, ni otra/ cosa alguna. Solamente en-/tonces auia Dios, que jamas / tuuo, ni tiene principio, ni te( $n) / \mathrm{dra}$ fin. Y quando le plugo, / y fue seruido, hizo y crio el / cielo, la tierra, y todo lo de-/mas que ay en ellos. Hizo el / cielo, para casa y morada de / los Angeles, y de los buenos / hombres. Hizo el sol para / dar resplandor y alumbrar el / dia. Ta $(m)$ bien crio la luna, ju $(n)$ ta-/ mente con las estrellas, para a/lu $(m)$ brar la noche, y darle clari-/dad. Hizo este mundo, para / que nosotros los hombres bi/uiessemos, anduuiessemos y / morassemos en el. Hizo el / ayre, para que respirassemos. // las aues, los pexes, y todo lo / demas que ay criado, todo lo / hizo y crio para nosotros los / hombres. Algunas cosas de-/llas crio, para $\mathrm{q}($ ue ) nosotros co-/miessemos. Otros para $\mathrm{q}(u e)$ nos / ayudassen y siruiessen en nue/stras necessidades. Otras pa-/ra que nos gozassemos y hol/gassemos en verlas. Crio as-/si mismo alla en el cielo muy / gra $(n)$ cantidad y muchos cria-/dos suyos, que llamamos ange-/les. Los quales ni tiene $(n)$ car/ne, ni huesso, no tienen cuer-/po, son [e]spiritus puros como / nuestras animas. Estos ange-/les que digo, no son como los / ho $(m)$ bres, son de otro genero, / y [e]specie que nosotros. Destos // angeles q $(u e)$ os he dicho, algu/nos fuero( $(n)$ buenos y guarda-/ro $(n)$ y obedesciero $(n)$ los manda-/mie $(n)$ tos $\mathrm{d}(e)$ dios, $\mathrm{cu}(m)$ plie $(n)$ do su vo/lu( $(n) \operatorname{tad}$. Y estos agora esta $(n)$ con / el en el cielo, y son bie $(n)$ aue $(n)$ tu-/rados, estando en gran conte $(n)$-/to y gloria, sin faltarles cosa / ninguna de las que dessean, / Y a estos llamamos Angeles / buenos. Otros fueron muy / malos, no obedesciero $(n)$ a dios, / ni guardaron sus mandamie $(n) /$ tos cu $(m)$ pliendo su volu $(n) \operatorname{tad}$, 
an-/tes $\operatorname{peccaro}(n)$ y enojaro(n) mucho / a dios $\mathrm{n}($ uest)ro señor. $\mathrm{Y}$ a estos por / sus peccados, los echo Dios / del cielo, y desterro aca baxo / de la tierra, al infierno en gra $(n)$ / fuego, y obscuridad, y hedor //Do( $n)$ de hasta agora esta $(n)$, y esta-/ra $(n)$ para sie $(m)$ pre encerrados, pa-/descie $(n)$ do por sus peccados. Y / estos son los $\mathrm{q}($ ue $)$ en $\mathrm{v}($ uest)ra lengua / llamays (mana alli çupay.) Y / nosotros en la nuestra, les lla-/mamos diablos. Despues $\mathrm{q}(u e) /$ dios ouo hecho y criado to-/das estas cosas $\mathrm{q}(u e)$ os he dicho. / Crio en este $\mathrm{mu}(n)$ do vn ho $(m)$ bre / llamado Adam, y vna muger / llamada Eua. Y d $(e)$ ste ho $(m)$ bre, $\mathrm{y} / \mathrm{d}(e)$ sta muger, nosotros los chri/stianos, y vosotros los indios, / y todos los negros, y los in-/dios d(e) Mexico, y los indios $\mathrm{q}($ ue $) /$ esta(n) en los mo(n)tes, y todos $\mathrm{q}($ uan $) /$ tos ho $(m)$ bres ay derramados y / diuididos del vn cabo d $(e) 1 \mathrm{mu}(n) / \mathrm{do}$ hasta el otro, todos $\mathrm{d}(e)$ ellos // $\mathrm{p}($ ro $)$ cedemos. Y nacemos. Y este / ho $(m)$ bre llamado Adam, y esta / muger llamada Eua, son $\mathrm{n}($ uest)ro / principio, y de do $(n)$ de procede/mos. Y dellos, $\mathrm{n}($ uest)ros antepassa/dos $\mathrm{p}($ ro $)$ cediero $(n)$, y se fuero $(n)$ a mo/rar a España, donde nosotros / biuimos. Y v(uest)ros antepassados / $(\mathrm{d}(e)$ quie $(n)[\mathrm{es}]$ vosotros venis $)$ vinie/ro $(n)$ a biuir a esta $\mathrm{t}($ ie)rra, do $(n)$ de ago/ra estays. Y los antepassados / $\mathrm{d}(e)$ los negros se fuero $(n)$ a sus tier/ras. Y los antepassados de los Mexicanos, y assi mismo los / de todos los ho( $m)$ bres $\mathrm{q}($ ue $)$ esta $(n)$ di/uididos por todo el $\mathrm{mu}(n)$ do, se / diuidiero $(n)$ por diuersas $\mathrm{t}($ ie)rras. / Aueys $\mathrm{d}(e)$ saber $\mathrm{q}($ ue $) \mathrm{aq}(u e)$ llos demo/nios $\mathrm{q}(u e)$ os dixe, $\operatorname{te}(n) \operatorname{tar}(n)$ a $\mathrm{n}($ uest $)$ ros $\mathrm{p}($ ri $) /$ meros padres, $\mathrm{y}$ diero $(n) \operatorname{ocasio}(n) / /$ tentandolos para que pecas/sen, $\mathrm{y}$ assi peccaro(n). Y estos de/monios son los q(ue) a nosotros / cada día nos aco(n)sejan el pec-/car, engañandonos y persua-/diendonos lo malo, y a voso-/tros (aunque no los veys) os / pone $(n)$ en vuestros coraçones / malos pe $(n)$ samientos, os dize $(n)$, / Adorad al sol, a la luna, a las / piedras, a los ydolos. Y, por / esto, aueys enojado $\operatorname{co}(n) \mathrm{v}($ uest $)$ ros pe/cados mucho a dios n(uest)ro se-/ñor. Por esso auisad de aqui a/delante, y no lo hagays assi / como hasta agora, sino de a-/qui adela(n)te eme $(n)$ daos de vue-/stros peccados, y co $(n)$ vuestros / coraçones y pensamientos a-/llegaos a dios nuestro señor, // diziendo, O señor mio, vos soys mi señor y cria-/dor. Hasta agora no os he conoscido, y assi (adora $(n)$ do los ydolos) os he mucho enojado. De aqui ad/elante me emendare, y nunca mas peccare. Y a / vos solo adorare y amare, mas que a todas las co=/sas. Biuiendo assi, y siendo christianos, quando / murieredes, vuestras animas yra $(n)$ al cielo $\operatorname{co}(n)$ Dios / para siempre jamas. Amen. 


\section{ANNEXE C}

\section{LA CONFESSION GENERAL}

Yo muy gran peccador me co(n)fesso, y di/go todos mis peccados a Dios, y a san/ cta Maria, y a sancto Domingo, y a to=/dos los sanctos, y a vos padre, que he pecca/do mucho en mal pensar, en mal hablar, ha=/blando en vano, comiendo y beuiendo de=/ masiado mal obrando riyendo hazie $(n)$ do bur/la de otros, andando en balde, jugando, jura $(n) /$ do $\operatorname{sie}(n)$ do negligente y perezoso en bie $(n)$ obrar. / Por tanto, de todos ellos mis peccados me / pesa y me eme $(n)$ dare dellos, y no boluere mas / a peccar. Y ruego a señora sancta Maria vir/gen, y madre de Dios, y a todos los sanctos / Rueguen a Dios por mi, y a vos padre ro=/gueys a Dios por mi, y en su nombre me $a b=/$ soluare dellos. Amen.

\section{La confession general, en la / lengua Indiana}

Nóca ancha hochallicócmi, appo diósta, sancta Maria/cta, sancto Domingocta, llapa Diospa yananta, hó=/chayta villani. $\mathrm{Ca}(\mathrm{m})$ padrectapas hochallicúscayta vil/ llayqui. Nanac hochallicurcani, mana allicta rimaspa, cacimanta rimaspapas yalli/spa micuspa, yallispa vpiaspapas, mana allicta ruraspa, / accispa (sic), accipayaspa (sic) (1). yanga porispa, yanga pucllaspa/alli cacta rurangaypac quecllacuspa ( sic) (2) Chayrayco, cay / llapa hochallicuscayma $(n)$ ta llaquipuni, guanassacmi / ñispa. Mana ñatac hochallicussacchu, Señora sancta / Maria tazqui diospa mamanta llapa diospa yananc=/ guantac mochani, pay appo dios ruraquenchicta/ñoca hochallicocpac mochapuanganpac, cam / padrepas appo dióspa randin cac, hochalli=/cuscaymanta quispichiuay. / Amen.

\section{La confesión general en la lengua indiana}

Ñuqa, ancha huchallikuqmi, apu Diosta, Santa Maríakta, Santo Domingokta, llapa Diospa yananta, huchayta willani. Qam, padrektapaŝ huchallikuŝqayta willayki. Nanaq huchallikurqani, mana allikta rimaŝpa qasimanta rimaŝpapaŝ, yalliŝpa mikuŝpa, yalliŝpa upyaŝpapaŝ, mana allikta ruraŝpa, asiŝpa, asipayaŝpa, yanqa puriŝpa, yanqa pukllaŝpa, alli kaqta ruranqaypaq qillakuŝpa. Chayrayku, kay llapa huchallikuŝaymanta llakipuni “wanaŝaqmi” ñiŝspa. Mana ñataq huchallikuŝaqchu. Señora Santa María, taski Diospa mamanta llapa Diospa yananwantaq muchani, pay (3) apu Dios ruraqinchikta ñuqa huchallikuqpaq muchapuwanqanpaq. Qam padrepaŝ, apu Diospa rantin kaq, huchallikuŝaymanta qiŝspichiway. Amen.

\section{Traduction française de la Confession Générale}

Moi, plein de péchés, je confesse mes péchés à [Notre] Seigneur Dieu, à Sainte Marie, à Saint Dominique, à tous les anges (4) de Dieu. Et à toi, mon père, je confesse

(1) ¿Lapsus pour acispa et acipayaspa?

(2) ¿quellacuspa? Cependant, on trouve la forme quecllani comme variante de quillacuni dans le Lexicon, p. 356.

(3) Emploi démonstratif de pay.

(4) Lit. "tous les serviteurs". 
ce que j'ai péché. J'ai péché très gravement en disant du mal et en racontant des choses sans fondement, en mangeant et en buvant à l'excès, en faisant de mauvaises actions, en me comportant de manière frivole (5), en me moquant des autres, en perdant mon temps dans des promenades et des jeux, en négligeant l'accomplissement de bonnes oeuvres. Pour ce motif, je regrette profondément mes péchés et j'ai l'intention de me corriger. Je ne pécherai plus. J'invoque (6) la Vierge, Notre Dame Sainte Marie, mère de Dieu ainsi que tous les anges (7) de Dieu afin qu'ils intercèdent en ma faveur, moi pécheur, auprès de Dieu, l'auteur de nos jours. Et toi, mon père, représentant de [Notre] Seigneur Dieu, délivre-moi de mes péchés. Amen.

(5) Lit. "en riant". C'est-à-dire, en ne pensant qu'à m'amuser au lieu de me consacrer à des tâches profitables pour le bien de mon âme et de la société coloniale.

(6) Généralement, dans les textes d'évangélisation, mucha- se traduit par "adorer". Nous basons notre traduction ici sur le contexte. De la même manière, plus loin dans le même énoncé, nous traduisons le même lexème par "intercéder".

(7) Lit. "tous les serviteurs". 